Collection SFN 10 (2010) 563-588

(C) Owned by the authors, published by EDP Sciences, 2010

DOI: $10.1051 / \mathrm{sfn} / 2010014$

\title{
Transitions de phase structurales
}

\author{
R. Currat
}

Institut Laue-Langevin, 6 rue Jules Horowitz, BP. 156X, 38042 Grenoble, France

\begin{abstract}
We review the characteristic features of the two main categories of structural phase transitions (displacive and order/disorder), with respect to static properties (order parameter) as well as dynamical aspects (critical fluctuations). We describe of few examples of displacive transitions (ferroelectric and antiferrodistortive transitions in perovskites, incommensurate phases in dielectrics, low-D metals and molecular crystals), with emphasis on the dynamical aspects (soft mode, central peak) and on the relevant experimental techniques, inelastic neutron scattering in particular.
\end{abstract}

Résumé. On rappelle les caractéristiques principales des deux catégories de transitions de phase structurales (displacives et ordre/désordre), du point de vue des propriétés statiques (paramètre d'ordre) et dynamiques (fluctuations critiques). On décrit quelques exemples de transitions displacives (transitions ferroélectrique et antiferrodistortive dans les pérovskites, phases incommensurables dans les diélectriques, les métaux de basse dimensionnalité et les cristaux moléculaires), en mettant l'accent sur les aspects dynamiques (mode mou, pic central) et sur les techniques expérimentales qui permettent leur étude, la diffusion inélastique des neutrons, notamment.

\section{INTRODUCTION : TRANSITIONS DISPLACIVES ET ORDRE/DÉSORDRE}

Il existe une grande variété de transitions structurales entre phases cristallines. On se concentrera ici sur les transitions continues ou faiblement discontinues, pour lesquelles il existe une phase de haute symétrie, stable à haute température - si c'est la température qu'on fait varier expérimentalement pour passer d'une phase à l'autre - et une phase de basse symétrie stable à basse température. Si c'est la pression qui varie, alors on aura des cas où la phase de haute symétrie sera la phase stable à haute pression - c'est le cas par exemple pour les transitions paraélectrique $\Leftrightarrow$ ferroélectrique - ou l'inverse [1].

Plus précisément, le caractère continu de la transition implique que le groupe d'espace de la phase de basse symétrie - on va dire basse température (BT) pour simplifier - est un sous-groupe [2] du groupe d'espace de le phase HT. Il existe donc une quantité mesurable, qui apparaît en dessous de la température de transition $\mathrm{T}_{\mathrm{c}}$, et qui réduit la symétrie de la phase HT. Certains éléments de symétrie vont disparaître, ceux qui subsistent constituant le groupe d'espace de la phase BT.

Cette quantité mesurable, on va l'appeler le paramètre d'ordre (PO) de la transition et suivant la nature physique de ce $\mathrm{PO}$ on va distinguer deux grandes catégories de transitions structurales, les transitions du type ordre/désordre (O/D) et les transitions du type displacif.

Les transitions displacives sont caractérisées soit par des déformations de la maille cristalline, on les appelle alors transitions ferroélastiques, soit par des déplacements atomiques au sein de la maille avec, dans un cas comme dans l'autre, une brisure de symétrie. La Fig. 1(a) montre l'exemple d'un cristal cubique de structure $\mathrm{CsCl}$ avec des atomes de type $\mathrm{A}$ aux coins du cube élémentaire et un atome de type $B$ au centre avec, en dessous de $T_{c}$, un déplacement du sous-réseau B par rapport au sous-réseau A.

This is an Open Access article distributed under the terms of the Creative Commons Attribution-Noncommercial License 3.0, which permits unrestricted use, distribution, and reproduction in any noncommercial medium, provided the original work is properly cited. 


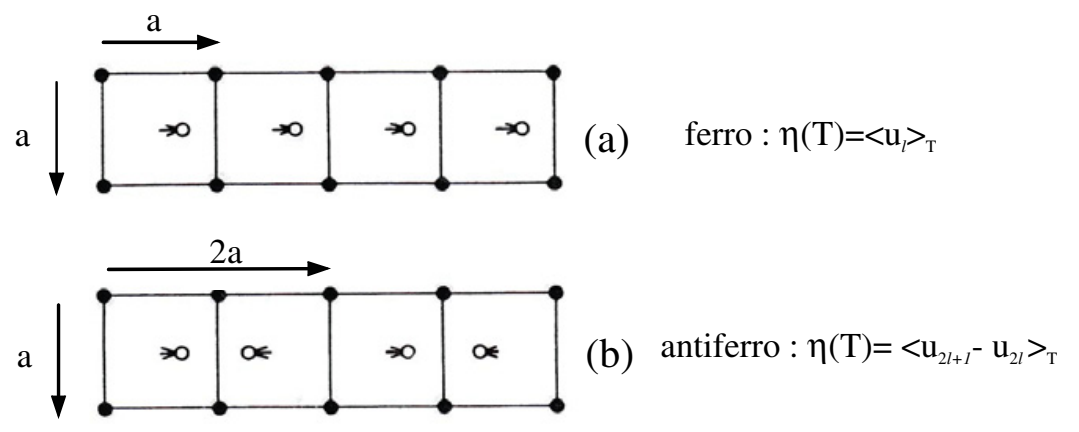

Figure 1. Exemple de transitions displacives (a) «ferro» et (b) «antiferro» dans un cristal cubique de structure $\mathrm{CsCl}$.

Le paramètre d'ordre dans ce cas, sera la moyenne statistique des déplacements atomiques : moyennés dans le temps, puisque les atomes vibrent et on parle ici, de positions atomiques moyennes, et moyennés sur les mailles. Le PO, $\eta$, est a priori une fonction de la température. C'est clair dans le cas d'une transition continue, puisque $\eta$ doit être nul à $\mathrm{T}_{\mathrm{c}}$ et croître continûment en dessous de $\mathrm{T}_{\mathrm{c}}$. C'est vrai également pour les transitions faiblement discontinues.

On peut aussi avoir des déplacements alternés de maille à maille (cf. Fig. 1(b)) et, dans ce cas, le PO sera défini comme la moyenne des déplacements atomiques dans les mailles impaires moins la moyenne des déplacements dans les mailles paires. On aura un doublement du paramètre de maille dans la direction d'alternance des déplacements atomiques et par conséquent de nouvelles raies de Bragg - les raies de surstructure - vont apparaître en dessous de $\mathrm{T}_{\mathrm{c}}$, dont l'intensité va croître approximativement comme le carré des déplacements atomiques, i.e. le carré du PO.

Dans un cas comme dans l'autre on aura des domaines, i.e. des variétés différentes de la même phase BT (6 domaines dans les 2 cas ci-dessus), le nombre de domaines étant directement relié au nombre d'éléments de symétrie perdus.

En général il y a plusieurs quantités qui apparaissent simultanément à $\mathrm{T}_{\mathrm{c}}$ et, parmi elles, on identifie le 'vrai' PO, ou PO principal, comme étant celui qui brise à lui tout seul le plus d'éléments de symétrie. Par exemple, dans les 2 cas «ferro» (Fig. 1(a)) et « antiferro » (Fig. 1(b)), il va apparaître une déformation tétragonale de la maille. La symétrie cubique étant brisée par les déplacements atomiques dans la direction z, la maille cristalline va relaxer, et il n'y a pas de raison physique pour que le paramètre de maille dans cette direction reste le même que le paramètre dans les 2 autres directions - ou égal exactement au double.

Dans le cas ferro, les déplacements font perdre, entre autres, les plans miroirs perpendiculaires à $\mathrm{z}$ et réduisent le groupe ponctuel de $\mathrm{m} 3 \mathrm{~m}\left(\mathrm{~T}>\mathrm{T}_{\mathrm{c}}\right)$ à $4 / \mathrm{mm}\left(\mathrm{T}<\mathrm{T}_{\mathrm{c}}\right)$, tandis qu'une simple déformation tétragonale de la maille, sans déplacement relatif des sous-réseaux, conserverait ces plans miroirs et conduirait à un groupe ponctuel tétragonal plus élevé, $4 / \mathrm{mmm}$. La déformation tétragonale ne fait donc pas partie du PO principal.

Dans le cas antiferro, les déplacements alternés conservent une partie des plans miroirs perpendiculaires à la direction $\mathrm{z}$ (ceux qui contiennent les atomes de type A), et le groupe ponctuel est donc 4/mmm, le même que dans le cas d'une simple déformation tétragonale. Mais les déplacements alternés, en plus, doublent la maille, i.e. qu'ils brisent une symétrie translationnelle, ce qui évidemment n'est pas le cas de la déformation tétragonale.

Dans les 2 cas, la déformation tétragonale apparaît donc comme un PO secondaire, induit par le PO principal.

L'autre catégorie de transitions de phase structurales est constituée par les transitions de type ordre/désordre. On prendra ici l'exemple des halogénures d'ammonium, qui sont parmi les premiers 
systèmes présentant un désordre orientationnel simple, à avoir été étudiés en détail [3-5]. Dans ces composés, les ions $\mathrm{NH}_{4}$ sautent entre 2 configurations possibles qu'on peut baptiser (A) et (B). Le schéma en Fig. 2 montre une des 2 configurations, pour laquelle les $\mathrm{H}$ pointent en direction de 4 des 8 halogènes aux sommets du cube élémentaire, les 4 autres halogènes étant concernés par l'autre configuration.

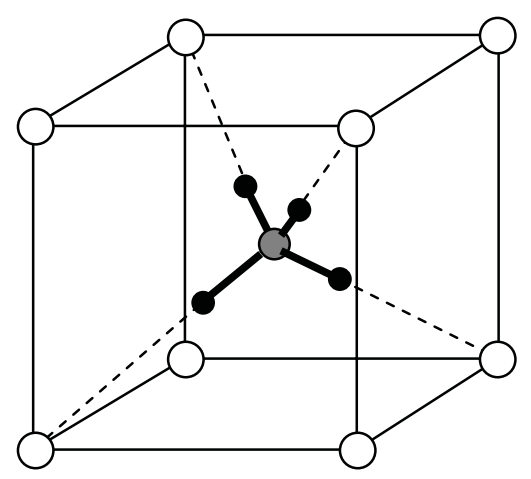

Figure 2. Structure des halogénures d'ammonium. En phase désordonnée, l'ion $\mathrm{NH}_{4}$ au centre du cube saute entre 2 configurations équivalentes (réorientations de $\pi / 2$ autour des axes $\langle 100\rangle$ ).

Il y a, en plus, des sauts possibles à l'intérieur d'une même configuration, par exemple des sauts de $2 \pi / 3$ autour des axes $\langle 111\rangle$, i.e. des diagonales du cube, et ces sauts donneront un signal en diffusion incohérente quasi-élastique des neutrons, mais pas en diffusion cohérente, car en diffusion cohérente le neutron se comporte comme une onde: il ne 'voit' pas le mouvement des particules individuelles, mais seulement l'évolution des configurations des centres diffuseurs [6].

Au-dessus de la température d'ordre, les 2 configurations (A) et (B) sont également probables, pour une maille donnée. Ce qui ne veut pas dire que le désordre est complet : évidemment, il y aura des corrélations entre les orientations des $\mathrm{NH}_{4}$ voisins, i.e. il y aura un certain degré d'ordre à courte portée, mais pas d'ordre à longue portée.

En dessous de la température d'ordre, les ions ammonium continuent de sauter gaiement d'une configuration à l'autre, mais localement, la moyenne dans le temps montre qu'une des 2 configurations est privilégiée. La configuration privilégiée n'est pas forcément la même partout, mais il y en a toujours une des 2 qui est localement plus probable et c'est l'écart de cette probabilité par rapport à la valeur $1 / 2$ qu'on va définir comme le PO.

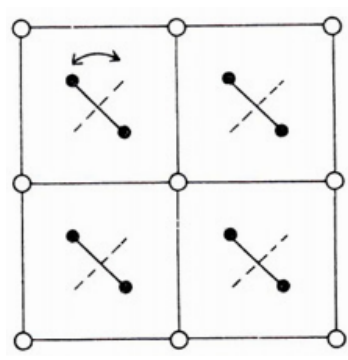

(a)

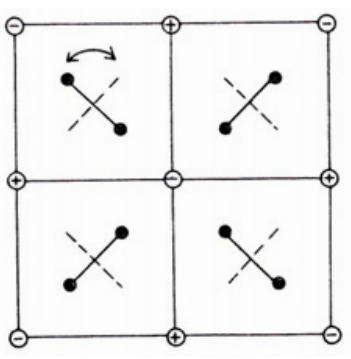

(b)

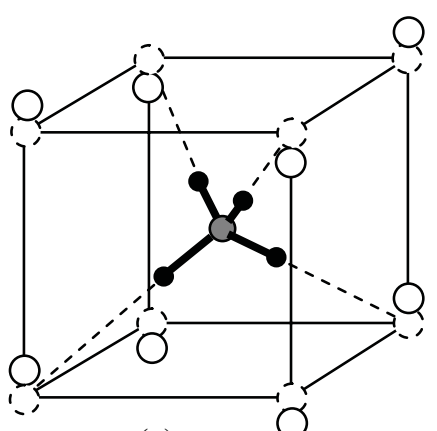

(c)

Figure 3. Halogénures d'ammonium: (a) ordre «ferro» $\left(\mathrm{NH}_{4} \mathrm{Cl}, \mathrm{T}_{\mathrm{c}}=242 \mathrm{~K}\right)$; (b, c) ordre partiellement «antiferro » $\left(\mathrm{NH}_{4} \mathrm{Br}, \mathrm{T}_{\mathrm{c}}=236 \mathrm{~K}\right)$. 
La distribution spatiale des configurations privilégiées peut être du type «ferro », i.e. identique dans toutes les mailles, c'est le cas de $\mathrm{NH}_{4} \mathrm{Cl}$ (Fig. 3(a)). Ici on montre une couche de 4 mailles et les traits pleins représentent l'arête supérieure des tétraèdres d'hydrogène, l'arête inférieure étant montrée en pointillés. Les configurations dans les couches successives sont toutes identiques et dans ce cas la structure reste cubique, avec un groupe ponctuel plus bas que dans la phase HT, puisqu'on a perdu des plans miroirs.

On peut également avoir un ordre du type partiellement «antiferro » comme dans $\mathrm{NH}_{4} \mathrm{Br}$, où les configurations alternent à l'intérieur de chaque couche, mais sont identiques d'une couche à l'autre (Fig. 3(b)). Dans ce cas, on obtient une structure de symétrie tétragonale puisque la direction z normale à la couche devient différente des directions $\mathrm{x}$ et $\mathrm{y}$.

On pourrait aussi imaginer un ordre complètement «antiferro » où les configurations alterneraient suivant les 3 directions $\mathrm{x}$, y et $\mathrm{z}$, ce qui donnerait encore une structure cubique, mais une telle structure n'est pas réalisée en pratique, dans ce type de composés, pour des raisons qui sont simples à comprendre si on s'intéresse, comme nous allons le faire à présent, à l'environnement local des ions halogènes.

Si on regarde ce qui se passe au niveau des sites des halogènes, par exemple celui qui est au centre du dessin en Fig. 3(a) et sur la face supérieure du cube, on voit qu'il est attiré électrostatiquement par les $2 \mathrm{H}$ de la couche inférieure qui vont donc le 'tirer' vers le bas, mais aussi par $2 \mathrm{H}$ de la couche supérieure qui vont le tirer vers le haut, et donc, dans ce cas l'halogène restera centré en position tétraédrique.

Par contre, dans le cas du composé au brome (Fig. 3(b)), on voit que le Br va être tiré vers le bas par $4 \mathrm{H}$, alors que dans la couche supérieure, tous les $\mathrm{H}$ des ions $\mathrm{NH}_{4}$ voisins pointent ailleurs et par conséquent dans ce cas, on observera un déplacement alterné des Br suivant z (Fig. 3(c)).

$\mathrm{Si}$ on analyse la perte de symétrie associée aux déplacements des $\mathrm{Br}$, on voit qu'à eux seuls ces déplacements brisent les mêmes éléments de symétrie que l'ordre orientationnel: ils font donc partie du $\mathrm{PO}$ principal et on a, dans ce cas, une transition de phase à caractère mixte $\mathrm{O} / \mathrm{D}$-displacif.

On comprend aussi pourquoi la structure complètement «antiferro » n'existe pas : on aurait alors des $\mathrm{Br}$ qui serait entourés de $8 \mathrm{H}$ et d'autres, d'aucun, et on imagine facilement que du point de vue électrostatique une telle situation soit énergétiquement défavorable.

\section{ASPECTS DYNAMIQUES ET FLUCTUATIONS CRITIQUES}

\subsection{Cas O/D}

Intéressons-nous à présent à ce qui se passe dans la phase dite désordonnée, lorsqu'on approche de la température d'ordre, $\mathrm{T}_{\mathrm{c}}$. D'une façon très générale, on s'attend à observer le développement d'agrégats (clusters) préfigurant la phase ordonnée, ou plus exactement, une des variantes de la phase ordonnée, puisque, dans le cas O/D comme dans le cas displacif, à partir du moment où il y a brisure de symétrie, la phase ordonnée pourra se présenter sous forme de plusieurs domaines différents. Dans le cas «ferro » qui est le plus simple, on aura des domaines où c'est la config. A qui est privilégiée et d'autres où c'est la config. B.

Le développement de ces agrégats est inévitable si on veut pouvoir passer continûment d'une phase à l'autre. Il faut donc s'attendre à voir la taille et la durée de vie moyennes de ces agrégats croître - et diverger à $\mathrm{T}_{\mathrm{c}}$, si la transition est véritablement continue.

De même, lorsqu'on approche la température de transition à partir de la phase ordonnée, dans un domaine donné, on observera la croissance d'agrégats correspondant aux autres domaines, agrégats dont la taille et la durée de vie moyennes divergeront à $\mathrm{T}_{\mathrm{c}}$ pour une transition continue.

Dans le cas des halogénures d'ammonium, le comportement des corrélations orientationnelles entre les ions $\mathrm{NH}_{4}$ peut se décrire formellement en introduisant une variable locale de spin, un pseudo-spin scalaire $\sigma(l)$ auquel on affectera la valeur \pm 1 selon que l'ion $\mathrm{NH}_{4}$ est en config. A ou B. 
En particulier, l'énergie d'interaction entre ammonium voisins pourra se mettre sous une forme analogue à une énergie d'échange spin-spin :

$$
\mathrm{V}=-1 / 2 \sum_{l l^{\prime}} \mathrm{J}_{l l^{\prime}} \sigma(l) \sigma\left(l^{\prime}\right)
$$

où les pseudo-intégrales d'échange $\mathrm{J}_{l l^{\prime}}$ représentent la partie du potentiel de paire $\mathrm{NH}_{4}-\mathrm{NH}_{4}$ qui dépend des orientations relatives des ions.

Par conséquent, on pourra, en principe, obtenir toutes les informations désirées sur l'état du système à chaque température, en transposant les résultats relatifs au modèle d'Ising. Pour ce qui concerne la diffusion cohérente des neutrons par exemple, la fonction de diffusion $S(\mathbf{Q}, \omega)$ s'exprime à partir du facteur de structure $\mathrm{F}(\mathbf{Q}, \mathrm{t})$ :

$$
S(\mathbf{Q}, \omega)=\int\langle\mathrm{F}(\mathbf{Q}, \mathrm{t}) \mathrm{F}(\mathbf{Q}, 0)\rangle \mathrm{e}^{\mathrm{i} \omega \mathrm{t}} \mathrm{dt}
$$

$\mathrm{F}(\mathbf{Q}, \mathrm{t})$ représente la somme des amplitudes diffusées à l'instant t par tous les atomes du système. C'est donc une double somme sur les mailles let sur les atomes dans la maille $\kappa$ :

$$
\mathrm{F}(\mathbf{Q}, \mathrm{t})=\sum_{l \kappa} \mathrm{b}_{\kappa} \exp \{\mathrm{i} \mathbf{Q} \cdot \mathbf{R}(l \kappa, t)\}
$$

Dans le cas de $\mathrm{NH}_{4} \mathrm{Cl}$, la somme sur les atomes de la maille s'écrira normalement pour les ions $\mathrm{Cl}$ et $\mathrm{N}$ et, pour les $\mathrm{H}$, ou plutôt pour les $\mathrm{D}$ puisqu'on parle de diffusion cohérente, on aura un facteur de structure moyen, $\mathcal{F}_{0}$, égal à la demi-somme des facteurs de structure des $\mathrm{D}$ en config. A et $\mathrm{B}$ :

$$
\mathrm{F}(\mathbf{Q}, \mathrm{t})=\sum_{l} \exp \{\mathrm{i} \mathbf{Q} \cdot \mathbf{R}(l)\}\left\{\mathrm{b}_{C l}+\mathrm{b}_{N} \exp \left(\mathrm{i} \mathbf{Q} \cdot \mathbf{R}_{\mathrm{N}}\right)+\mathcal{F}_{0}(\mathbf{Q})+\Delta \mathcal{F}(\mathbf{Q}) \cdot \sigma(l, \mathrm{t})\right\}
$$

et une quantité $\Delta \mathcal{F}$ qui, elle, mesure le contraste entre les 2 config.:

$$
\mathcal{F}_{0}=1 / 2\left\{\mathcal{F}_{\mathcal{A}}+\mathcal{F}_{\mathcal{B}}\right\} ; \Delta \mathcal{F}=1 / 2\left\{\mathcal{F}_{\mathcal{A}}-\mathcal{F}_{\mathcal{B}}\right\} ; \mathcal{F}_{\mathcal{A}, \mathcal{B}}=\sum_{\mathrm{D}} \mathrm{b}_{\mathrm{D}} \exp \left\{\mathrm{i} \mathbf{Q} \cdot \mathbf{R}_{\mathrm{D}}(\mathrm{A}, \mathrm{B})\right\}
$$

et qui est multipliée par la variable de spin, $\sigma(l, \mathrm{t})$, de façon à obtenir au total, $\mathcal{F}_{\mathcal{A}}$ ou $\mathcal{F}_{\mathcal{B}}$ suivant la valeur de la variable de spin.

En injectant (2.4) dans l'expression (2.2) pour $\mathrm{S}(\mathbf{Q}, \omega)$, on voit que la diffusion cohérente des neutrons donne accès aux fonctions de corrélation spatio-temporelles spin-spin, ou, plus directement, aux fonctions de corrélation temporelles des variables $\sigma(\mathbf{q})$, transformées de Fourier des variables locales de spin $\sigma(l)$.

\subsection{Cas displacif}

\subsubsection{Mode mou}

Revenons pour l'instant au cas des transitions displacives, et posons nous la question de savoir comment on peut décrire les petits déplacements des positions atomiques moyennes qui apparaissent en dessous de la température critique. On sait que tout écart par rapport aux positions atomiques moyennes de la phase symétrique (HT) peut se décomposer en champ de déplacements de phonons, de phonons de la phase HT, évidemment. Autrement dit les déplacements associés aux phonons constituent une base complète permettant de décrire n'importe quel champ de déplacements atomiques [7].

Il s'agit de phonons statiques bien sûr, puisque, on parle ici de déplacements statiques des positions atomiques moyennes. Un phonon, statique ou dynamique, se décrit à l'aide d'un vecteur propre e(qj) qui représente la géométrie des déplacements à l'intérieur de la maille cristalline, d'un vecteur d'onde q qui fixe la relation de phase entre les déplacements dans les différentes mailles, d'un facteur général d'amplitude $Q(\mathbf{q j})$ et d'une fréquence propre $\omega(\mathbf{q j})$, égale à 0 dans le cas statique. 
Le cas le plus simple est celui où les déplacements associés au PO principal peuvent être décrits à l'aide d'une seule coordonnée normale de phonon, de vecteur d'onde $\mathbf{q}_{\mathrm{s}}$ et d'indice de branche $\mathrm{j}_{\mathrm{s}}$. Si on reprend l'exemple du cristal cubique diatomique, on voit qu'il y aura certainement un phonon optique de centre de zone qui correspond aux déplacements indiqués Fig. 1(a). En fait, pour être précis, il faut rajouter un déplacement du sous-réseau $\mathrm{A}$ (Fig. 4(a)) parce qu'un mode optique à $\mathrm{q}=0$ conserve le centre de masse de la maille. Implicitement, on avait pris l'origine sur le sous-réseau A, mais en fait ce choix d'origine était arbitraire. La description en termes de déplacements de phonons montre que, dans ce cas, l'origine des déplacements doit être liée au centre de masse de la maille.

De même dans le cas «antiferro», il existe un mode de bord de zone qui correspond aux déplacements indiqués. Savoir s'il est sur la branche acoustique ou sur la branche optique, dépendra des masses des ions A et B et des valeurs des constantes de force [8].

Il y a évidemment des cas plus compliqués où plusieurs coordonnées normales de phonons peuvent être impliquées. Dans ce cas, on montre par des arguments très généraux de symétrie [2], que ces coordonnées normales ne peuvent pas être arbitraires, qu'elles appartiennent toutes à une même représentation irréductible du groupe d'espace de la phase symétrique, et qu'elles constituent, dans leur ensemble, une base complète de cette représentation irréductible. En particulier, elles sont toutes associées à une seule et même fréquence propre.

(a)

mode optique $\mathrm{q}=(0,0,0)$

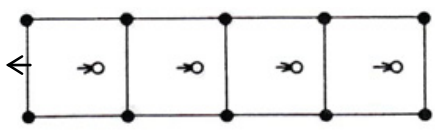

(b)

mode opt. ou ac. $\mathrm{q}=(0,0,1 / 2)$

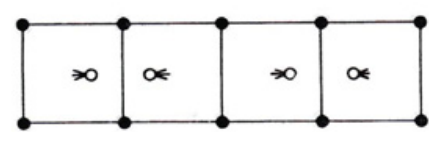

Figure 4. Déplacements atomiques et coordonnées normales de phonons : cas du cristal cubique diatomique.

La grande idée qui a été peu à peu confirmée par l'expérience et qui s'est donc imposée à partir des années 60 , consiste à faire l'hypothèse que l'analogie entre les déplacements statiques observés dans la phase BT et les déplacements de phonons de la phase HT, n'est pas seulement une analogie géométrique, mais qu'elle correspond à un mécanisme physique réel. Autrement dit, si des déplacements statiques de phonons apparaissent pour $\mathrm{T}<\mathrm{T}_{\mathrm{c}}$, c'est que la force de rappel associée à ce mode de phonon - ou à cet ensemble de modes de phonons dégénérés - doit s'annuler à $\mathrm{T}_{\mathrm{c}}$.

Et qui dit force de rappel dit fréquence propre et donc au-dessus de $\mathrm{T}_{\mathrm{c}}$ on doit s'attendre à observer un mode propre avec un comportement très fortement anharmonique, dont la fréquence doit tendre vers 0 quand $\mathrm{T} \rightarrow \mathrm{T}_{\mathrm{c}}$. C'est la théorie du mode mou, soft mode en anglais (d'où l'indice s qui apparaît partout par la suite), formulée indépendamment par Cochran [9] et Anderson [10].

Formellement, on écrira :

$$
\begin{gathered}
\mathrm{T}>\mathrm{T}_{\mathrm{c}} \Rightarrow \mathbf{u}_{l \kappa}\left(\mathbf{q}_{\mathrm{s}} \mathrm{j}_{\mathrm{s}}, t\right)=\left(\mathrm{Nm}_{\kappa}\right)^{-1 / 2} \mathrm{e}_{\kappa}\left(\mathbf{q}_{\mathrm{s}} \mathrm{j}_{\mathrm{s}}\right) Q\left(\mathbf{q}_{\mathrm{s}} \mathrm{j}_{\mathrm{s}}\right) \exp \left\{\mathrm{iq}_{\mathrm{s}} \mathbf{R}_{l \kappa}-i \omega\left(\mathbf{q}_{\mathrm{s}} \mathrm{j}_{\mathrm{s}}\right) t\right\}+\text { c.c. } \\
\left.\mathrm{T}<\mathrm{T}_{\mathrm{c}} \Rightarrow\left\langle\mathbf{u}_{l \kappa}\right\rangle=\left(\mathrm{Nm}_{\kappa}\right)^{-1 / 2} \mathrm{e}_{\kappa}\left(\mathbf{q}_{\mathrm{s}} \mathrm{j}_{\mathrm{s}}\right)\left\langle Q\left(\mathbf{q}_{\mathrm{s}} \mathrm{j}_{\mathrm{s}}\right)\right\rangle \exp \left\{\mathrm{iq}_{\mathrm{s}} \mathbf{R}_{l \kappa}\right)\right\}+ \text { c.c. }
\end{gathered}
$$

et on prend comme PO principal, l'amplitude moyennée statistiquement à la température T, de la coordonnée normale du phonon mou condensé (à un facteur de normalisation $\mathrm{N}^{-1 / 2}$ près) :

$$
\eta\left(\mathrm{T}<\mathrm{T}_{\mathrm{c}}\right)=\mathrm{N}^{-1 / 2}\left\langle Q\left(\mathbf{q}_{\mathrm{s}} \mathrm{j}_{\mathrm{s}}\right)\right\rangle_{\mathrm{T}}
$$




\subsubsection{Lois d'échelle}

A toute transition de phase continue est associée une échelle de temps et une échelle de longueur qui divergent au point critique et on a vu au $\$ 2.1$ que, pour les transitions de phase de type O/D, ces échelles correspondent au temps de vie et à la taille des régions corrélées. Que deviennent ces notions dans le contexte des transitions displacives et des théories de type mode mou?

D'abord, s'il y a une fréquence propre $\omega\left(\mathbf{q}_{\mathrm{s}} \mathrm{j}_{\mathrm{s}}\right)$ qui tend vers 0 , l'amplitude quadratique moyenne des fluctuations associées à ce ou ces modes va croître et en principe diverger à $\mathrm{T}_{\mathrm{c}}$ :

$$
\left\langle Q\left(\mathbf{q}_{\mathrm{s}} \mathrm{j}_{\mathrm{s}}\right) Q^{*}\left(\mathbf{q}_{\mathrm{s}} \mathrm{j}_{\mathrm{s}}\right)\right\rangle \equiv\left\langle Q\left(\mathbf{q}_{\mathrm{s}} \mathrm{j}_{\mathrm{s}}\right) Q\left(-\mathbf{q}_{\mathrm{s}} \mathrm{j}_{\mathrm{s}}\right)\right\rangle=\left\{\hbar / 2 \omega\left(\mathbf{q}_{\mathrm{s}} \mathrm{j}_{\mathrm{s}}\right)\right\}\left\{2 \mathrm{n}\left(\mathbf{q}_{\mathrm{s}} \mathrm{j}_{\mathrm{s}}\right)+1\right\} \approx \mathrm{kT} / \omega^{2}\left(\mathbf{q}_{\mathrm{s}} \mathrm{j}_{\mathrm{s}}\right)
$$

D'autre part, on doit prendre en compte le fait que le mode mou représente un point sur une courbe de dispersion de phonons, et que si sa fréquence décroît, le ramollissement doit concerner également les modes de vecteurs d'onde voisins sur cette courbe de dispersion, et ceci à un degré d'autant plus élevé que le vecteur d'onde $\mathbf{q}$ du mode est plus proche du vecteur d'onde $\mathbf{q}_{\mathbf{s}}$ du mode mou.

Si on développe la dispersion de la branche du mode mou au voisinage de $\mathbf{q}_{\mathbf{s}}, \mathbf{q}_{\mathbf{s}}$ étant nécessairement un minimum, le $1^{e r}$ terme du développement sera quadratique par rapport aux composantes de $\delta \mathbf{q}=$ $\mathbf{q}-\mathbf{q}_{\mathrm{s}}:$

$$
\omega^{2}\left(\mathbf{q} j_{\mathrm{s}}\right)=\omega^{2}\left(\mathbf{q}_{\mathrm{s}} \mathrm{j}_{\mathrm{s}}\right)+\sum_{\alpha} \lambda_{\alpha} \delta \mathrm{q}_{\alpha}^{2}+\cdots . \quad\left(\mathrm{T}>\mathrm{T}_{\mathrm{c}}\right)
$$

ce qui conduit à une décroissance lorentzienne des amplitudes quadratiques moyennes de fluctuations en fonction de l'écart $\delta \mathbf{q}$ :

$$
\left\langle Q\left(\mathbf{q} j_{\mathrm{s}}\right) Q\left(-\mathbf{q j}_{\mathrm{s}}\right)\right\rangle \approx\left\{\mathrm{kT} / \omega^{2}\left(\mathbf{q}_{\mathrm{s}} \mathrm{j}_{\mathrm{s}}\right)\right\}\left\{1+\sum_{\alpha} \xi_{\alpha}^{2} \delta \mathrm{q}_{\alpha}^{2}\right\}^{-1}
$$

avec:

$$
\xi_{\alpha}=\lambda_{\alpha}^{1 / 2} / \omega\left(\mathbf{q}_{\mathrm{s}} \mathrm{j}_{\mathrm{s}}\right)
$$

On a donc une situation de type paquet d'onde, où l'étendue du paquet d'onde dans l'espace direct, i.e. la région où les modes pourront avoir une interférence constructive, est d'autant plus grande que le paquet d'onde est étroit en $\delta \mathbf{q}$. Ici la largeur en $\mathbf{q}$ du paquet d'onde est donnée par $1 / \xi_{\alpha}$, et donc la variable $\xi$ va représenter la longueur de corrélation des fluctuations critiques.

On obtient ainsi une échelle de temps, $\omega^{-1}\left(\mathbf{q}_{\mathrm{s}} \mathrm{j}_{\mathrm{s}}\right)$, et une échelle de longueur, $\lambda^{1 / 2} / \omega\left(\mathbf{q}_{\mathrm{s}} \mathrm{j}_{\mathrm{s}}\right)$, qui toutes deux divergent à la température critique.

\section{LIMITE O/D-DISPLACIF : LE MODÈLE DES DOUBLE-PUITS COUPLÉS}

Jusqu'ici on a présenté les transitions displacives et des transitions O/D comme 2 catégories bien distinctes de transitions de phase structurales. Nous allons à présent montrer qu'il s'agit en fait de 2 cas limites, que l'on peut décrire dans le cadre d'un modèle plus général.

Si l'on s'intéresse à nouveau au cas des halogénures d'ammonium, on notera que dans un modèle purement $\mathrm{O} / \mathrm{D}$, les vibrations de réseau et, tout particulièrement les librations des ions $\mathrm{NH}_{4}$, ne sont pas prises en compte. Ceci sous-entend qu'elles ne jouent aucun rôle dans le mécanisme de la transition. Cette hypothèse est justifiée dans la mesure où, au voisinage de $T_{c}$, les amplitudes librationnelles moyennes, dans une config. donnée, sont faibles par rapport aux amplitudes des sauts réorientationnels. Ce découplage existe également au niveau des échelles de temps, les fréquences de saut étant beaucoup plus faibles que les fréquences propres des modes de libration.

Il existe de nombreux systèmes où un tel découplage n'est pas justifié et où l'instabilité structurale peut être décrite aussi bien - ou aussi mal - dans le cadre d'un modèle displacif ou O/D. Sur le plan théorique, le modèle des double-puits couplés, initialement proposé par Aubry [11] et repris par de 
nombreux auteurs $[12,13]$, permet de décrire aussi bien les 2 cas limites que les cas intermédiaires. Nous en donnons ci-après une version simplifiée, inspirée du travail de Bruce [13].

Il s'agit d'un ensemble de particules identiques soumises à un potentiel local anharmonique, en forme de double-puits (DP), et qui interagissent élastiquement entre elles via des ressorts de raideur C qui cherchent à maintenir les particules voisines à la distance $\boldsymbol{a}$ les unes des autres, où $\boldsymbol{a}$ est la périodicité du système de DP.

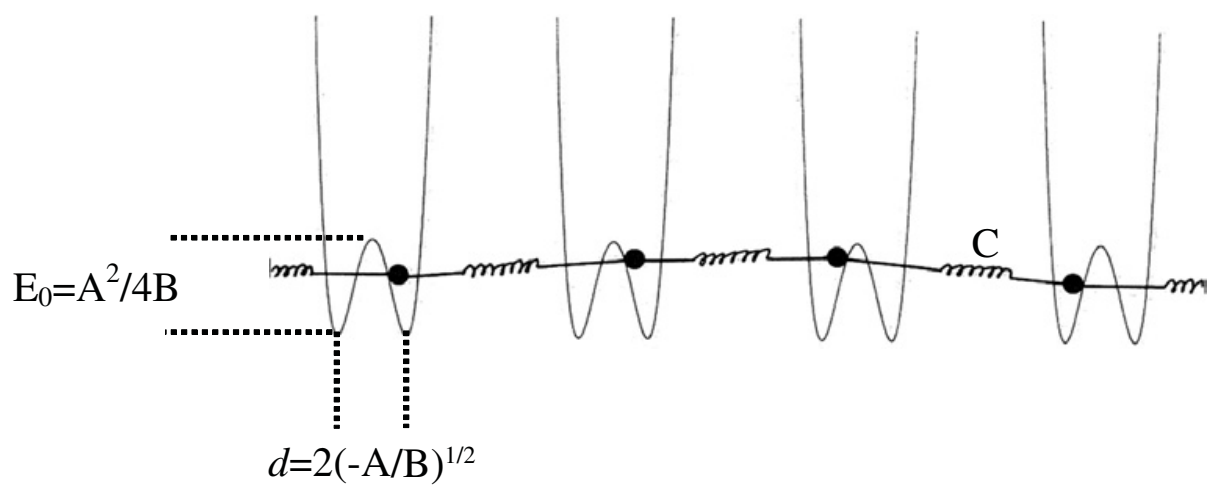

Figure 5. Le modèle des double-puits couplés [11-13].

Un tel système présente de toute évidence, une transition de phase - à température finie ou non, suivant sa dimensionnalité d'espace. À HT, les énergies thermiques sont grandes par rapport à la barrière de fond de puits $\mathrm{E}_{0}$ et par rapport aux énergies d'interaction entre particules, et donc les particules vont fluctuer de façon décorrélée, en explorant les 2 côtés de chaque DP. À BT par contre, à $0 \mathrm{~K}$ éventuellement, toutes les particules d'un même domaine, seront du même côté des DP (on considère des particules classiques). On a donc bien un changement de symétrie.

L'énergie potentielle du système s'écrit sous la forme :

$$
\mathrm{V}=\sum_{l} \mathrm{~V}_{0}\left(u_{l}\right)+\mathrm{C} / 2 \sum_{l, l^{\prime}}\left(u_{l}-u_{l^{\prime}}\right)^{2} ; \quad \mathrm{V}_{0}(u)=\mathrm{A} / 2 u^{2}+\mathrm{B} / 4 u^{4} ; \quad(\mathrm{A}<0)
$$

où la variable $u_{l}$ représente l'écart de position de la particule $l$ par rapport au milieu du DP. La barrière de fond de puits $\mathrm{E}_{0}$ et la distance entre les 2 puits $d$ s'expriment en fonction des paramètres $\mathrm{A}(<0)$ et $\mathrm{B}(>0)$ du potentiel local $\mathrm{V}_{0}$ (voir Fig. 5).

On écrit les équations de mouvement des particules en différenciant le potentiel :

$$
\mathrm{m} \ddot{u}_{l}+(\mathrm{A}+4 \mathrm{C}) u_{l}+\mathrm{B} u_{l}^{3}-2 \mathrm{C} \sum_{\text {n.n. }} u_{l^{\prime}}=0
$$

où la somme $\sum_{n . n}$ s'étend aux premiers voisins de la particule $l$.

On obtient ainsi $\mathrm{N}$ équations couplées. Comme on ne sait que faire du terme en $u_{l}^{3}$, on le linéarise: $u_{l}^{3} \approx u_{l}\left\langle u^{2}\right\rangle_{\mathrm{T}}$; approximation de Hartree). Le système d'équations linéaires ainsi obtenu peut ensuite être diagonalisé en introduisant les transformées de Fourier des variables locales $u_{l}$, i.e. les coordonnées normales de phonons $\mathrm{Q}_{\mathrm{q}}$.

C'est donc une approximation de type quasi-harmonique où on remplace le potentiel anharmonique par un potentiel harmonique effectif, qui va évidemment varier avec la T. Statistiquement parlant, c'est une approximation analogue à l'approximation de champ moyen, puisque, en linéarisant on découple les phonons, alors qu'en fait, ils sont couplés par le terme d'anharmonicité. Dans l'approximation du champ moyen habituelle, i.e. dans l'espace direct, on découple les particules qui sont en interaction. 
On réduit le problème à $\mathrm{N}$ corps à un problème de $\mathrm{N}$ particules indépendantes soumises au champ moyen des autres. On fait de même ici pour les phonons.

On obtient ainsi la loi de dispersion des phonons :

$$
\omega^{2}(q)=A+4 C(1-\cos q \mathrm{a})+\mathrm{B}\left\langle u^{2}\right\rangle_{\mathrm{T}}
$$

qui est du type optique (puisque le système des particules n'est pas invariant par translation), avec un minimum à $\mathrm{q}=0$, égal à $\mathrm{A}+\mathrm{B}\left\langle u^{2}\right\rangle_{\mathrm{T}}$.

À température suffisamment élevée, le terme positif $\mathrm{B}\left\langle u^{2}\right\rangle_{\mathrm{T}}$ compense le terme $\mathrm{A}(<0)$ et $\omega^{2}(0)>0$ : l'état du système est stable. Quand la température diminue, $\left\langle u^{2}\right\rangle_{\mathrm{T}}$ diminue et à un moment les 2 termes vont s'annuler: on aura alors une fréquence propre nulle, i.e. une instabilité structurale.

Pour savoir à quelle température l'instabilité aura lieu, il faut pouvoir évaluer le terme $\left\langle u^{2}\right\rangle_{\mathrm{T}}$ à chaque température. En principe $\left\langle u^{2}\right\rangle_{\mathrm{T}}$ est obtenu en sommant les amplitudes thermiques moyennes de tous les phonons du système. Or ces amplitudes sont déterminées par le spectre des fréquences propres des phonons (voir éq. (2.9)), lesquelles dépendent précisément de la valeur de $\left\langle u^{2}\right\rangle_{\mathrm{T}}$ :

$$
\left\langle u^{2}\right\rangle_{\mathrm{T}}=\mathrm{N}^{-1} \sum_{\mathrm{q}}\left\langle\mathrm{Q}_{\mathrm{q}} \mathrm{Q}_{-\mathrm{q}}\right\rangle \approx \mathrm{N}^{-1} \mathrm{kT} \sum_{\mathrm{q}} \omega^{-2}(\mathrm{q})=\mathrm{kT} \sum_{\mathrm{q}}\left\{\mathrm{A}+4 \mathrm{C}(1-\operatorname{cosqa})+\mathrm{B}\left\langle u^{2}\right\rangle_{\mathrm{T}}\right\}^{-1}
$$

L'équation (3.4) ci-dessus peut donc être considérée comme un critère de consistance interne. On notera en passant qu'un critère analogue apparaît dans le cas de l'approximation du champ moyen.

Si l'on suppose, pour simplifier, que la transition de phase est continue, la température de transition, $\mathrm{T}_{\mathrm{c}}$, coïncide avec celle de l'instabilité. En remplaçant $\left\langle u^{2}\right\rangle_{\mathrm{T}=\mathrm{Tc}}$ par $|\mathrm{A}| / \mathrm{B}$ dans (3.4), on obtient alors la valeur de $\mathrm{T}_{\mathrm{c}}$ :

$$
\mathrm{kT}_{\mathrm{c}}=4|\mathrm{~A}| \mathrm{C} / \mathrm{B} \mathscr{I}=\mathrm{C} d^{2} / \mathscr{I} ; \mathscr{I}=\mathrm{N}^{-1} \sum_{\mathrm{q}}(1-\operatorname{cosqa})^{-1}(\approx 0.5 \text { pour } \mathrm{D}=3)
$$

où la sommation sur la zone de Brillouin converge vers une valeur proche de 0.5 pour un système tridimensionnel.

L'expression (3.5) montre que $\mathrm{kT}_{\mathrm{c}}$ est de l'ordre de $\mathrm{C} d^{2}$, où $d$ est la distance entre les 2 fonds de puits, i.e. de l'ordre de l'énergie de couplage à dépenser pour déplacer une particule d'un fond de puits à l'autre, à basse température, lorsque le système est parfaitement ordonné.

Par ailleurs, il est clair que l'approximation quasi-harmonique ne sera acceptable que si, à $\mathrm{T}_{\mathrm{c}}$, l'énergie thermique des particules est suffisamment élevée pour leur permettre d'explorer les 2 côtés du DP de façon oscillatoire, ce qui impose $\mathrm{kT}_{\mathrm{c}}>\mathrm{E}_{0}$ et donc $\mathrm{C} d^{2}>\mathrm{E}_{0}$. C'est la limite displacive.

Que se passe-t-il dans la limite inverse, $\mathrm{C}^{2}<\mathrm{E}_{0}$ ? Lorsque $\mathrm{kT}$ sera de l'ordre de $\mathrm{E}_{0}$, les particules vont se localiser progressivement dans les fonds de puits avant que l'ordre ne s'établisse, i.e. de façon aléatoire et on aura une gamme de température, $\mathrm{C} d^{2}<\mathrm{kT}<\mathrm{E}_{0}$, où la structure sera désordonnée (au sens $\mathrm{NH}_{4} \mathrm{Cl}$ ) et c'est donc à plus basse température, lorsque $\mathrm{kT}$ sera de l'ordre de $\mathrm{C} d^{2}$ que l'ordre s'établira. C'est la limite O/D.

Une autre façon de définir le régime displacif [12], c'est de noter que, lorsque le système est parfaitement ordonné, si on change une particule d'un fond de puits à l'autre, l'énergie élastique emmagasinée $\left(\mathrm{Cd}^{2}\right)$ est suffisante pour que la particule revienne immédiatement à sa position d'origine dès qu'on la 'lâche' : la position énergétiquement défavorable est instable $\left(\mathrm{Cd}^{2}>\mathrm{E}_{0}\right)$.

Alors que dans le cas $\mathrm{O} / \mathrm{D}\left(\mathrm{C}^{2}<\mathrm{E}_{0}\right)$, la particule est piégée dans un état métastable et elle doit attendre une fluctuation thermique locale pour réintégrer sa position d'origine.

On voit que le modèle des double-puits couplés permet d'appréhender les rôles respectifs des potentiels locaux et des potentiels d'interaction entre particules. La transition de phase n'a lieu que si le potentiel local est du type double-puits. Par contre la température de transition, elle, est entièrement déterminée par les énergies d'interaction entre particules, dans le cas displacif comme dans le cas O/D. On remarquera cependant que dans les systèmes réels, la séparation entre potentiel interparticule et potentiel local n'est pas toujours évidente à faire en pratique. 


\section{EXEMPLES DE TRANSITIONS DISPLACIVES}

Nous passons à présent en revue quelques systèmes parmi les plus connus qui présentent des comportements displacifs plus ou moins bien caractérisés.

\subsection{Modes mous férroélectriques dans les pérovskites oxygénées}

Les transitions paraélectrique $\rightarrow$ ferroélectrique dans les pérovskites oxygénées, comme $\mathrm{BaTiO}_{3}$, $\mathrm{KNbO}_{3}$ et $\mathrm{PbTiO}_{3}$, ont fait l'objet de très nombreuses études expérimentales et théoriques.

$\mathrm{Si}$ on prend comme origine le réseau des barium - ce qui n'est pas le meilleur choix comme on l'a vu au \$2.2.1- on peut, en première approximation, décrire la transition comme un déplacement relatif des cages d'oxygène par rapport à l'ion central, ici le titane (Fig. 6).

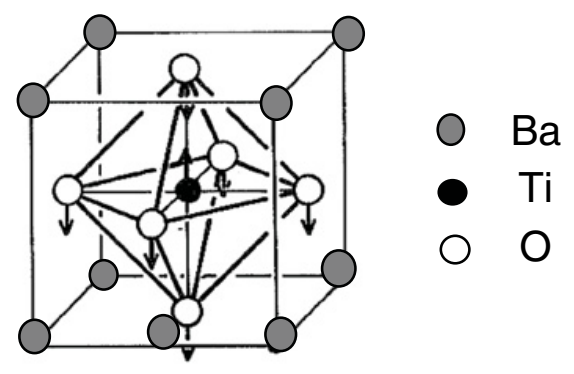

Figure 6. Transition cubique (paraél.) $\rightarrow$ tétrag. (ferroél.) dans $\mathrm{BaTiO}_{3}\left(\mathrm{~T}_{\mathrm{c}}=393 \mathrm{~K}\right)$. Le déplacement des $\mathrm{O}$ le long de z s'accompagne d'une légère déformation des cages d' $\mathrm{O}$, les $\mathrm{O}$ voisins de l'ion Ti le long de z, se déplacent plus que les autres.

Les ions étant chargés $\left(\mathrm{Ti}^{4+}, \mathrm{O}^{--}\right)$, un moment dipolaire électrique apparaît dans chaque maille, et comme les déplacements sont identiques dans toutes les mailles, on obtient un moment dipolaire macroscopique, et par conséquent la phase tétragonale est ferroélectrique.

Du point de vue de la dynamique, ces transitions ne peuvent se décrire de façon satisfaisante ni dans un modèle displacif, ni dans un modèle O/D. Il y a bien des modes mous, mais ils sont fortement amortis et très couplés anharmoniquemt avec les autres modes. Ce ne sont donc pas des bons exemples de transitions displacives.

On peut les décrire aussi comme des systèmes O/D. C'est le modèle de Comès, Lambert, Guinier [14], qui marche assez bien à condition de supposer que l'ion titane est soumis à un potentiel local pour lequel les «fonds de puits» sont décalés le long des directions $\langle 111\rangle$ par rapport au centre du cube. Ces directions correspondent aux centres des faces des octaèdres d'oxygène et on peut raisonnablement supposer que c'est le long de ces directions que l'ion central a la possibilité de se déplacer le plus librement.

Mais il faut aussi postuler l'existence de fortes corrélations entre les déplacements des Ti, ce qui ne va pas bien avec un modèle O/D. Ce sont des corrélations partielles, au sens où, certaines composantes des déplacements sont corrélées dans certaines directions et pas dans d'autres.

En fait, une des difficultés du problème est liée au fait que, malgré la symétrie cubique, il existe de très fortes anisotropies dans les dispersions des modes de phonon. Dans certaines directions, on observe des modes très dispersifs, indiquant un fort couplage entre particules compatible avec un modèle displacif, tandis que dans d'autres directions, on observe des modes très peu dispersifs, caractéristiques d'une situation où les potentiels locaux jouent un rôle prépondérant par rapport aux potentiels interparticule, comme dans un modèle de type O/D. Par conséquent, il n'existe pas de modèle simple permettant de décrire ces transitions de façon satisfaisante. 
Par contre, il existe d'autres composés ayant la même structure et qui présentent une tendance vers le même type d'instabilité, mais pour lesquels l'ion central a moins de place pour bouger: ce sont les incipient ferroelectrics, $\mathrm{KTaO}_{3}$ et $\mathrm{SrTiO}_{3}$. Ces 2 systèmes se comportent comme s'ils devaient transiter à basse température $(5-10 \mathrm{~K})$, mais, dans les 2 cas, la phase cubique est stabilisée par les fluctuations quantiques [15], qui, dans cette gamme de température, sont comparables en amplitude aux fluctuations thermiques.

$\mathrm{KTaO}_{3}$ et $\mathrm{SrTiO}_{3}$ présentent de beaux exemples de modes mous de centre de zone $(\mathrm{q}=0)$, avec des fréquences propres de modes qui varient d'un facteur 3 entre l'ambiante et $6 \mathrm{~K}$ (Fig. 7) et des largeurs de raies inférieures au 1/10 de la fréquence.

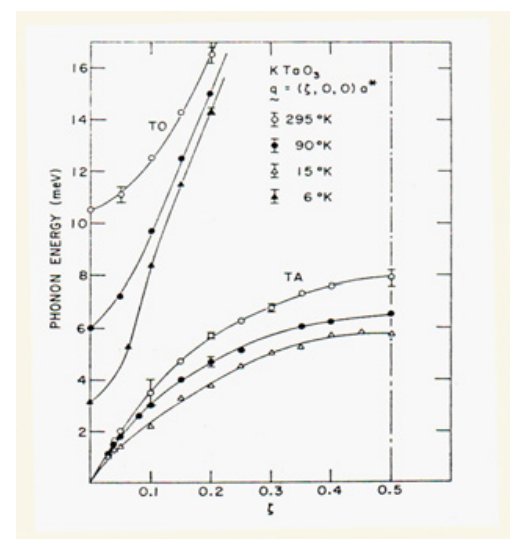

Figure 7. $\mathrm{KTaO}_{3}$ : dispersion du mode transverse acoustique (TA) et transverse optique (TO) dans la direction 100 (Axe et al., réf. [16]). L'anharmonicité de la branche TO induit un comportement anharmonique pour la branche TA. Le couplage entre les 2 branches est de nature harmonique. La pente à $\mathrm{q}=0$ de la branche TA (vitesse du son transverse) n'est pas affectée par le couplage et reste constante en fonction de la température. Le mode mou ferroélectrique est le mode TO à q = 0 .

La Fig. 8(a) montre l'accord parfait entre la fréquence propre du mode mou ferroélectrique mesurée en diffusion inélastique des neutrons par Farhi et al. (réf. [17]) et en spectroscopie hyper-Raman, par Voigt (réf. [18]). Le mode n'est pas actif en spectroscopie Raman normale, du fait que, en phase cubique, tous les ions sont en position centro-symétrique. Le mode est par contre actif en spectroscopie infrarouge puisqu'il génère un moment dipolaire oscillant (mode polaire). La dispersion des modes polaires est caractérisée par la présence d'un champ électrique dépolarisant dans le cas des modes LO (modes où le moment dipolaire oscillant est parallèle au vecteur d'onde), alors que pour les modes TO il n'y a pas de champ dépolarisant et donc pas de force de rappel supplémentaire. Cet écart en fréquence entre composantes TO et LO persiste dans la limite $\mathrm{q} \rightarrow 0$ du fait du caractère «longue portée » des interactions dipolaires. Le mode mou est donc toujours le mode TO le plus bas en fréquence (TO1).

La Fig. 8(b) montre la comparaison entre la fréquence propre carrée du mode mou et l'inverse de la constante diélectrique statique $1 / \varepsilon(0)$, les 2 étant reliés par la relation de Lyddane-Sachs-Teller [19] (LST). La fréquence propre du mode mou ferroélectrique reflète bien la quasi-divergence de $\varepsilon(0)$. La relation LST est un cas particulier d'une relation plus générale qui relie $1 /\left(\omega_{\mathrm{MODE} \text { MOU }}\right)^{2}$ à la susceptibilité statique associée au paramètre d'ordre [20], ici, la polarisation diélectrique spontanée.

\subsection{Modes mous en bord de zone de Brillouin : la transition antiferrodistortive de $\mathrm{SrTiO}_{3}$}

Une autre catégorie de transitions structurales très étudiée concerne les modes de bord de zone de Brillouin dans la même structure pérovskite de base. Contrairement au cas des transitions ferroélectriques, qu'on trouve uniquement dans les pérovskites oxygénées de formule $\mathrm{ABO}_{3}$, et ce, 

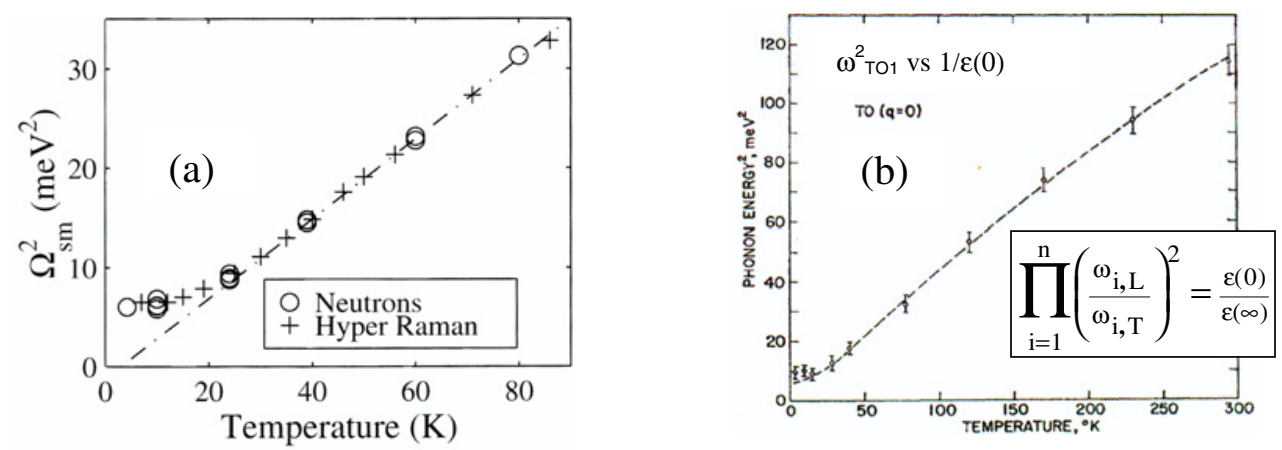

Figure 8. Mode mou ferroélectrique dans $\mathrm{KTaO}_{3}$ : (a) Variation en température de la fréquence propre carrée (d'aprés Farhi et al. (réf. [17]) ; (b) Illustration de la relation de Lyddane-Sachs-Teller entre $\omega_{\mathrm{TO} 1}^{2}$ et $1 / \varepsilon(0)$ (d'après Shirane et al. (réf. [21]).

du fait de la polarisabilité électronique [22] exceptionnellement élevée de l'ion $\mathrm{O}^{--}$, les modes mous de bord de zone, eux, se rencontrent aussi bien dans les pérovskites oxygénées que dans les composés au $\mathrm{F}$ ou au $\mathrm{Cl}$, comme $\mathrm{KMnF}_{3}, \mathrm{RbCaF}_{3}$ ou $\mathrm{CsPbCl}_{3}$, par exemple.

\subsubsection{Structures}

La transition vers $100-105 \mathrm{~K}$ du titanate de strontium, $\mathrm{SrTiO}_{3}$, est de très loin la mieux connue de toutes les transitions de ce type et même de toutes les transitions structurales, en général [23].

(a)

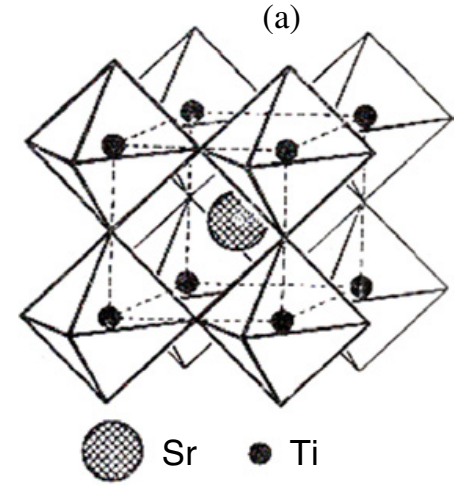

(b)

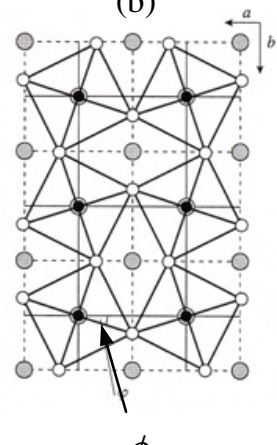

$\phi$

Figure 9. Structure perovskite $\left(\mathrm{SrTiO}_{3}\right)$ : (a) Phase cubique (b) Phase tétragonale : les octaèdres d'oxygène tournent d'un angle $\pm \phi$ autour de l'axe c, normal au plan de la figure. Le sens des rotations alterne suivant les 3 directions $(\mathbf{a}, \mathbf{b}, \mathbf{c})$.

$\mathrm{Si}$ on prend en compte le fait que les octaèdres d'oxygène $(\mathrm{OO})$ sont des unités relativement compactes et rigides - même si $\mathrm{Ti}^{4+}$ peut, dans certains cas (voir §4.1) se déplacer hors du centre on voit (Fig. 9(a)) qu'il y a beaucoup de place libre entre les OO. Il est possible de rendre la structure plus compacte en faisant pivoter les OO sur eux-mêmes de façon alternée (Fig. 9(b)) : on force ainsi les centres des $\mathrm{OO}$, les $\mathrm{Ti}^{4+}$, de se rapprocher, ce qui a pour effet de tasser la structure. A basse température, les amplitudes moyennes de vibration des ions, et par conséquent le volume qu'ils occupent, diminuent. Plutôt que de se contracter de façon homogène, vu la rigidité des $\mathrm{OO}$, la structure pourra choisir de faire 
pivoter les OO sur eux-mêmes. L'important, dans ce cas, c'est essentiellement les valeurs des rayons ioniques et en particulier celui du $\mathrm{Sr}^{++}$, qui joue le rôle d'espaceur entre les OO. Comme on peut s'y attendre, la température de transition augmente fortement sous pression [24].

Si on suppose que les OO tournent de façon parfaitement rigide autour de la direction c (Fig. 9(b)), les angles de rotation alternent nécessairement $(+\phi,-\phi$, etc..) pour la couche d'OO dans le plan $(\mathbf{a}, \mathbf{b})$. Au niveau des fluctuations critiques, on peut donc prévoir de fortes corrélations planaires dans le plan $(\mathbf{a}, \mathbf{b})$, i.e. un mode mou fortement dispersif dans le plan $\left(\mathbf{a}^{*}, \mathbf{b}^{*}\right)$. Par contre le long de c, il n'y a pas de raison structurale évidente de nature à fixer la relation de phase entre les rotations dans un plan et les rotations dans les plans voisins. On aura donc une dispersion assez plate dans cette direction.

Cette relative indétermination se reflète dans les structures ordonnées elles-mêmes, puisqu'on trouve des composés qui s'ordonnent de façon partiellement «antiferro», avec des rotations identiques dans les couches successives. C'est le cas pour $\mathrm{KMnF}_{3}$ à basse température $(\mathrm{T}<91 \mathrm{~K})$, avec un vecteur d'onde critique situé au point M 1/2(110) de la zone de Brillouin cubique [25]. Dans d'autres systèmes, tels $\mathrm{SrTiO}_{3}, \mathrm{RbCaF}_{3}, \mathrm{LaAlO}_{3}$ ainsi que $\mathrm{KMnF}_{3}(91 \mathrm{~K}<\mathrm{T}<186 \mathrm{~K})$, la structure ordonnée est du type «antiferro» avec des rotations alternées d'un plan à l'autre et donc des transitions au point R 1/2(111) de la zone de Brillouin (Fig. 10(a)).

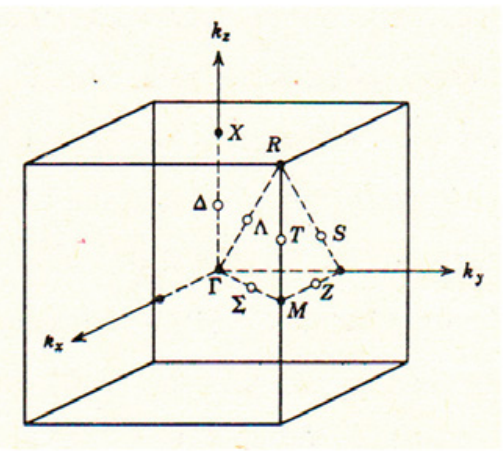

(a)

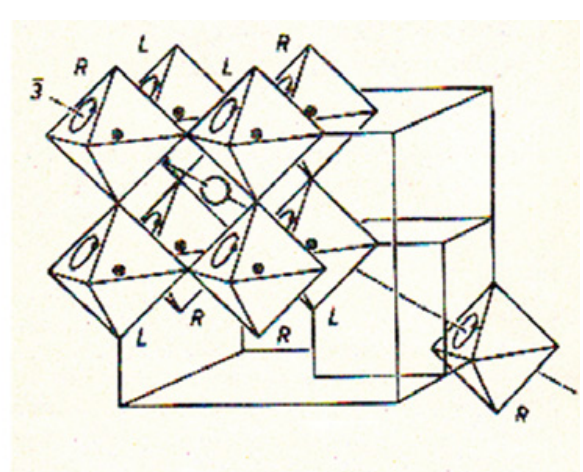

(b)

Figure 10. (a) Zone de Brillouin de la structure pérovskite cubique ; (b) $\mathrm{LaAlO}_{3}$ (phase trigonale): rotations alternées des octaèdres d'oxygène autour des axes $\langle 111\rangle$.

Au point $\mathrm{R}$, le mode de rotation des $\mathrm{OO}$ est triplement dégénéré au sens où on peut choisir de pivoter autour des directions [100], [010] ou [001]. D'ailleurs, en combinant des rotations élémentaires autour de ces 3 directions, on peut générer des rotations autour d'un axe arbitraire : on a un espace vectoriel de vecteurs propres de dimension 3, à l'intérieur duquel on peut choisir une base arbitraire. Il se trouve que dans la plupart des cas, on observe des rotations autour les axes $\langle 100\rangle$, et des phases BT de symétrie tétragonale, mais des rotations de type $\langle 111\rangle$ sont observées dans le composé $\mathrm{LaAlO}_{3}$ (Fig. 10(b)), ce qui conduit à une phase BT de symétrie trigonale [26]. Le nombre de domaines possibles sera de $3 \times 2=6$ dans le cas $\langle 100\rangle$ et de $4 \times 2=8$ dans le cas $\langle 111\rangle$. On peut aussi induire des rotations $\langle 111\rangle$ dans le $\mathrm{SrTiO}_{3}$ en appliquant une contrainte uniaxiale le long d'une direction $\langle 111\rangle$ [27,28], avec, dans ce cas, seulement les 2 domaines de type antiphase $(+\phi,-\phi \Leftrightarrow-\phi,+\phi)$.

Pour les transitions au point M 1/2(110), on a 2 domaines d'antiphase par point $\mathrm{M}$ et 6 points $\mathrm{M}$ distincts, soit 12 domaines au total.

Les rotations alternées des $\mathrm{OO}$ entraînent un doublement de la maille cristalline et par conséquent l'apparition de raies de surstructure dont l'intensité varie comme le carré du PO, $\langle\phi\rangle^{2}$. La mesure de l'intensité de ces raies, à l'aide des RX ou des neutrons (Fig. 11(a)) permet donc en principe d'accéder à 
la variation en température du PO. Toutefois, l'expérience montre que de telles mesures sont entachées d'imprécisions d'origine multiple :

- problèmes d'extinction loin de $\mathrm{T}_{\mathrm{c}}$ quand l'intensité de la raie à mesurer devient importante.

- incertitudes liées au facteur de Debye-Waller, qui présente une anomalie [29] de type cusp au voisinage de $\mathrm{T}_{\mathrm{c}}$, due à la contribution de la branche du mode mou aux amplitudes quadratiques moyennes de fluctuation des ions - des $\mathrm{O}$ dans le cas présent.

- et surtout le fait qu'on mesure une contribution supplémentaire, inélastique ou quasiélastique, associée aux fluctuations critiques (mode mou ou autre), et ce, aussi bien pour $\mathrm{T}<\mathrm{T}_{\mathrm{c}}$ que pour $\mathrm{T}>\mathrm{T}_{\mathrm{c}}$. D'où des difficultés pour déterminer la valeur précise de $\mathrm{T}_{\mathrm{c}}$ et pour séparer les 2 contributions en dessous de $\mathrm{T}_{\mathrm{c}}$.
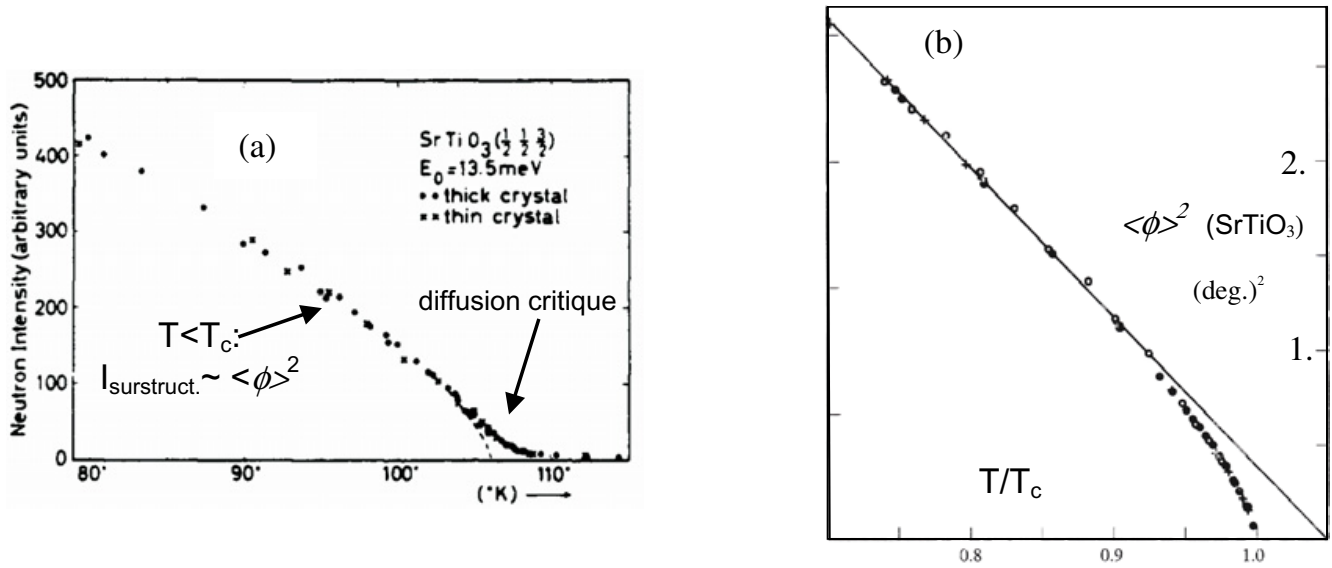

Figure 11. $\mathrm{SrTiO}_{3}$ : Détermination de la variation en température du paramètre d'ordre (a) par diffraction des neutrons (Riste et al., réf. [29]) ; (b) par RPE (Müller et Berlinger, réf. [30]).

Le même type d'information est accessible à l'aide des techniques de résonance (RPE, RQN, RMN). L'effet des fluctuations critiques est moins important dans ce cas, puisque toutes les fluctuations plus rapides que le temps caractéristique de la mesure sont moyennées à zéro, le temps caractéristique se situant dans la gamme du $\mathrm{MHz}$ ou du $\mathrm{KHz}$, suivant la technique utilisée. Il est clair que, en diffusion des neutrons, et a fortiori des RX, l'utilisation d'un analyseur (config. «3-axes ») ne permet pas un filtrage aussi efficace des composantes inélastique ou quasiélastique des intensités mesurées. Les mesures RPE du PO de $\mathrm{SrTiO}_{3}$ par Müller et Berlinger [30] sont parmi les plus précises qui existent, pour une transition de phase structurale (Fig. 11(b)).

\subsubsection{Dynamique}

La Fig. 12 montre la dispersion du mode mou de bord de zone $\left(\mathrm{R}_{25}\right)$, le long de la direction [111] ( $\Gamma$-R en Fig. 10(a)). On notera la forte dispersion des branches acoustique et optique le long de cette direction, ce qui suggère une variation rapide des vecteurs propres des modes. L'anti-croisement de branches vers $1.1 \AA^{-1}$ correspond à un échange de vecteurs propres entre les branches acoustique et optique. Le mode optique mou de centre de zone $\left(\Gamma_{15}\right)$ correspond au mode ferro-électrique du $\$ 4.1$.

La Fig. 13(a) montre la variation en température de la fréquence du mode $\mathrm{R}_{25}$, mesurée en phase cubique $\left(\mathrm{T}>\mathrm{T}_{\mathrm{c}}\right)$ par diffusion inélastique des neutrons. On observe un comportement de type mode mou, même si la fréquence du mode n'est pas strictement nulle à $\mathrm{T}_{\mathrm{c}}$. Nous reviendrons sur ce point au $\S 5$. 


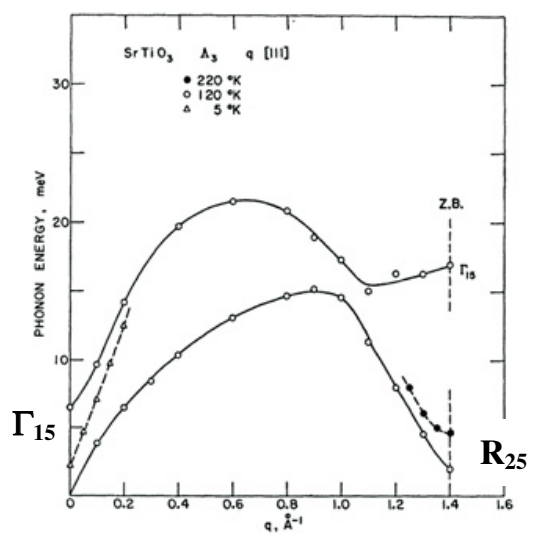

Figure 12. $\mathrm{SrTiO}_{3}$ : Dispersion des branches TA et TO1 le long de la direction [111]. $\mathrm{R}_{25}$ : rotation alternée des octaèdres d'oxygène autour d'un axe arbitraire ; $\Gamma_{15}:$ mode mou ferroélectrique (d'après Shirane et al., (réf. [31]).

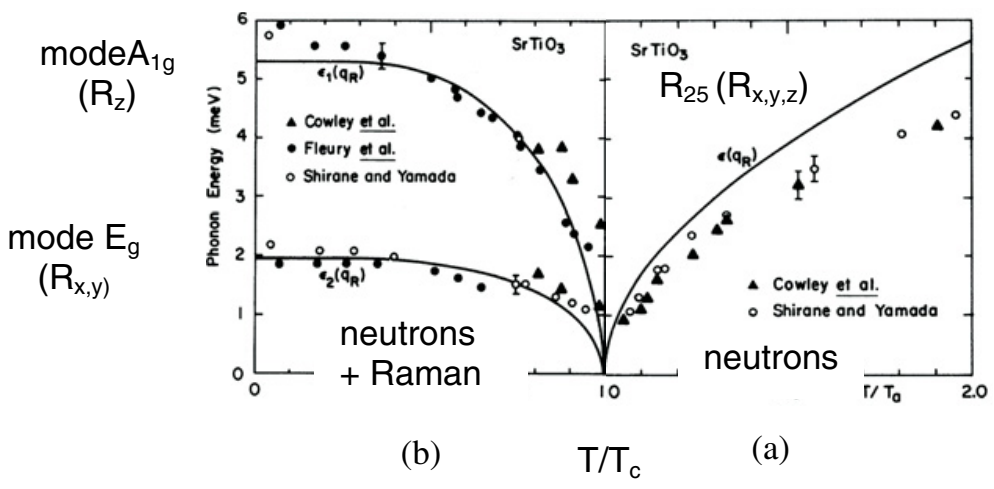

Figure 13. Mode mou $\mathrm{R}_{25}$ dans $\mathrm{SrTiO}_{3}$ : variation en température de la fréquence propre (a) $\left.\mathrm{T}\right\rangle \mathrm{T}_{c}$; (b) $\mathrm{T}\left\langle\mathrm{T}_{c}\right.$. Data neutrons et Raman resp., d'après réfs. [31,32] et [33]; $(-)$ : modèle théorique d'après réf. [34].

En dessous de $\mathrm{T}_{c}$ les $\mathrm{OO}$ vibrent autour des nouvelles positions d'équilibre, décalées de $\pm \phi_{\mathrm{z}}$, par rapport aux positions cubiques (on suppose que les rotations ont lieu autour de la direction z). Dans le langage des doubles-puits couplés du §3, on dira que les $\mathrm{O}$ se localisent progressivement dans les fonds de puits.

$\mathrm{A} \mathrm{T} \ll T_{\mathrm{c}}$, le mode de vibration des $\mathrm{OO}$ autour de la direction $\mathrm{z}, \mathrm{R}_{\mathrm{z}}$, correspond donc à la vibration de fond de puits. Sa fréquence est donnée par la (forte) courbure du potentiel local au fond du puits, d'où la fréquence élevée du mode $\mathrm{R}_{\mathrm{Z}}$ dans la limite des BT (Fig. 13(b)).

Quel comportement peut-on prédire pour le mode doublement dégénéré, $\mathrm{R}_{\mathrm{xy}}$, correspondant aux vibrations des $\mathrm{OO}$ autour des directions $(\mathrm{x}, \mathrm{y})$ ? Comme il n'y a pas eu de rotation statique autour de ces directions, on pourrait très bien concevoir que la fréquence du mode $\mathrm{R}_{\mathrm{xy}}$ reste nulle à toutes températures $\mathrm{T}<\mathrm{T}_{\mathrm{c}}$.

Ce n'est pas le cas ici, parce que les rotations d'OO autour des directions $\mathrm{x}$, y et $\mathrm{z}$ sont indépendantes seulement au premier ordre, i.e. seulement pour des rotations de faible amplitude, du type phonon. Mais il existe des termes d'ordre plus élevé dans l'énergie du système qui couplent les rotations (x,y,z) entre elles, et ces termes doivent être pris en compte lorsqu'on considère des rotations statiques d'amplitude finie. 
Dans $\mathrm{SrTiO}_{3}$, ces termes sont répulsifs, i.e. que des rotations simultanées autour de 2 ou 3 axes de type $\langle 100\rangle$ sont énergétiquement défavorables. C'est la raison qui fait que le système choisit des rotations $\langle 100\rangle$. Lorsque ces termes sont attractifs, comme dans $\mathrm{LaAlO}_{3}$, les 3 rotations $\langle 100\rangle$ se condensent simultanément avec des amplitudes égales, ce qui équivaut à une rotation de type $\langle 111\rangle$. C'est ce même mécanisme qui stabilise ici le mode doublement dégénéré $\mathrm{R}_{\mathrm{xy}}$ en présence d'une rotation statique $\left\langle\mathrm{R}_{\mathrm{z}}\right\rangle$ d'amplitude finie et qui lui donne une fréquence faible mais non nulle, proportionnelle à $\left\langle\mathrm{R}_{\mathrm{z}}\right\rangle$.

Au-dessus de $\mathrm{T}_{\mathrm{c}}$, seuls les neutrons, et dans une certaine mesure les RX, permettent d'étudier le comportement du mode de bord de zone $\mathrm{R}_{25}$. Au premier ordre, les techniques optiques (diffusion Raman, absorption infrarouge) ne voient que les phonons de centre de zone $(\mathrm{q}=0)$ et sont donc inefficaces dans ce cas.

En dessous de $T_{c}$, les 2 modes $R_{z}\left(A_{1 g}\right)$ et $R_{x y}\left(E_{g}\right)$ deviennent actifs en diffusion Raman parce que le point $\mathrm{R}$ de la zone de Brillouin cubique devient un centre de zone en phase tétragonale, du fait du doublement de la maille. Une autre manière de raisonner consiste à dire qu'on a toujours la même structure et la même zone de Brillouin et qu'on s'intéresse à présent à la diffusion Raman à 2 phonons (Raman du $2^{\text {ième }}$ ordre), où l'on mesure la somme ou la différence des fréquences des 2 phonons qu'on absorbe ou qu'on excite simultanément [35]. Dans ce cas, les 2 phonons doivent avoir des vecteurs d'onde égaux et opposés de façon à ce qu'au total on ait un transfert de moment nul $(\mathrm{Q} \approx 0)$.

$\mathrm{Si}$ on prend les déplacements alternés statiques comme un phonon, de fréquence nulle et de vecteur d'onde $1 / 2$ (111), et qu'on l'associe à un des phonons au point $\mathrm{R}, \mathrm{R}_{\mathrm{xy}}$ ou $\mathrm{R}_{\mathrm{z}}$ par exemple, on voit qu'on a un mécanisme qui permet, en principe, d'activer ces modes en diffusion Raman, en dessous de $T_{c}$. C'est d'ailleurs de cette façon qu'on calcule les règles de sélection qui contrôlent les conditions d'observabilité de l'ensemble des modes qui deviennent optiquement actifs dans la phase BT [36].

\subsection{Phases incommensurables}

Il n'y a pas d'interdiction à ce qu'une instabilité structurale se développe pour un vecteur d'onde qui ne soit pas un point de haute symétrie de la zone de Brillouin, i.e. un centre de zone ou un centre de face de zone. Ces vecteurs d'onde de haute symétrie peuvent s'écrire :

$$
\mathbf{q}_{\mathbf{S}}=1 / 2 \mathbf{G}_{\mathbf{h k l}} \equiv 1 / 2\left(h \mathbf{a}^{*}+k \mathbf{b}^{*}+l \mathbf{c}^{*}\right) ; \quad(h, k, l: \text { entiers })
$$

où $\mathbf{G}_{\mathbf{h k l}}$ est un vecteur de translation du réseau réciproque HT. Dans la plupart des cas, ces points de HS sont tels que les courbes de dispersion des phonons (et des électrons) sont stationnaires avec une pente, i.e. une vitesse de groupe, nulle. C'est donc des points privilégiés pour un minimum de dispersion de type mode mou, mais, en principe, un minimum peut intervenir accidentellement n'importe où à l'intérieur ou sur la surface de la zone de Brillouin.

C'est le cas en particulier pour les transitions de phase incommensurables pour lesquelles le vecteur d'onde critique s'écrit :

$$
\mathbf{q}_{\mathrm{S}}=\xi \mathbf{a}^{*}+\eta \mathbf{b}^{*}+\zeta \mathbf{c}^{*}
$$

où au moins un des nombres $(\xi, \eta, \zeta)$ n'est pas un rationnel simple (par exemple $\xi \neq \mathrm{n} / \mathrm{m}$ avec $\mathrm{m}<10$ ).

\subsubsection{Diélectriques incommensurables : $\mathrm{K}_{2} \mathrm{SeO}_{4}$}

La Fig. 14 montre la dispersion du mode TA $\left(\Sigma_{3}\right)$ se propageant dans la direction [100], pour le composé $\mathrm{K}_{2} \mathrm{SeO}_{4}$, en phase orthorhombique. En fait la figure montre 2 courbes de dispersion représentées séparément pour plus de clarté.

Les 2 courbes sont dégénérées au pt X $(1 / 2,0,0)$ avec des pentes égales et opposées. Tout se passe comme si le cristal avait une périodicité égale à $\mathrm{a} / 2$ et donc une zone de Brillouin double dans la direction [100]. 
Ceci provient du fait que le groupe d'espace contient un plan de glissement associé à une translation fractionnaire $\mathbf{a} / 2$. Ceci implique que, entre 2 plans cristallins identiques espacés de $\mathbf{a}$, il existe un plan intermédiaire à la position $\mathbf{a} / 2$, dont la structure atomique se déduit de la structure des 2 autres plans par une opération de symétrie miroir.

Les modes de phonon qui se propagent le long de a ne 'voient' pas cette différence de structure et par conséquent la périodicité cristalline leur apparaît plus élevée qu'elle ne l'est en réalité. On dit encore qu'il existe un invariant de Lifshitz [38] au point X et il est clair que, dans ce cas, l'instabilité ne pourra pas avoir lieu au point $\mathrm{X}$.

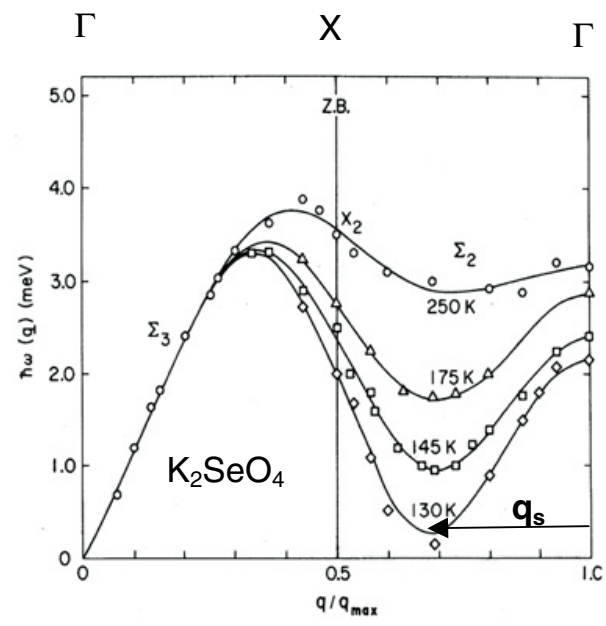

Figure 14. $\mathrm{K}_{2} \mathrm{SeO}_{4}$ : Courbe de dispersion de la branche du mode mou en schéma de zone étendue (Axe et al., réf. [37]).

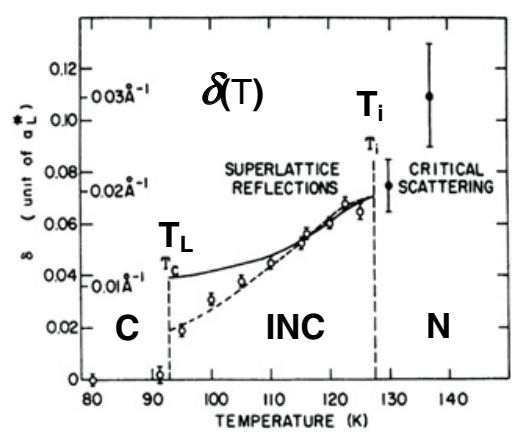

Figure 15. $\mathrm{K}_{2} \mathrm{SeO}_{4}$ : Variation du paramétre d'incommensurabilité $\delta(\mathrm{T})$; d'après Iizumi et al. (réf. [39]).

Ici le minimum de la dispersion, et donc le vecteur d'onde critique, se situe au voisinage de 1/3 (2/3 sur la figure) :

$$
\mathbf{q}_{\mathrm{s}}=\mathbf{a}^{*} / 3\{1-\delta(\mathrm{T})\}
$$

Le paramètre $\delta$ pointe la position du maximum de la diffusion critique au-dessus de la température de transition $T_{i}=129 \mathrm{~K}$, et la position des raies de surstructure (ou raies satellites) en dessous de $\mathrm{T}_{\mathrm{i}}$, i.e. la périodicité spatiale des déplacements atomiques modulés. Sa valeur dépend du détail des constantes 
de force interatomiques et peut donc varier en fonction de la température ou de n'importe quel autre champ ou paramètre externe (Fig. 15).

À BT $\left(\mathrm{T}<\mathrm{T}_{\mathrm{L}}=94 \mathrm{~K}\right)$, la structure incommensurable devient instable vis-à-vis de la structure modulée commensurable de période $3 \mathrm{a}(\delta=0)$.

La nature et le mécanisme de cette seconde transition de phase à $\mathrm{T}_{\mathrm{L}}$ ( $\mathrm{L}$ pour lock-in), sont très différents de le transition entre phase cristalline normale et phase incommensurable à $\mathrm{T}_{\mathrm{i}}$. En effet, on peut montrer [39] que l'énergie libre du cristal modulé contient des termes qui favorisent une valeur commensurable pour la période $2 \pi / \mathrm{q}_{\mathrm{s}}$ des déplacements atomiques. Ces termes sont toutefois d'ordre élevé (d'ordre 6 dans le cas présent) par rapport à l'amplitude des déplacements atomiques, dont la valeur moyenne thermique constitue le PO de la phase modulée. L'importance relative des termes de lock-in croît en dessous de $\mathrm{T}_{\mathrm{i}}$, à mesure que l'amplitude moyenne des déplacements augmente. Les termes de lock-in deviennent dominants pour $\mathrm{T}<\mathrm{T}_{\mathrm{L}}$. Ils influencent également le comportement en température du paramètre $\delta(\mathrm{T})$ pour $\mathrm{T}_{\mathrm{L}}<\mathrm{T}<\mathrm{T}_{\mathrm{i}}$.

\subsubsection{Transitions de Peierls dans KCP}

Dans les diélectriques incommensurables, tels $\mathrm{K}_{2} \mathrm{SeO}_{4}$, la position du vecteur d'onde critique dans la zone de Brillouin, n'a pas de signification microscopique particulière. On peut la considérer comme un minimum accidentel sur la branche de phonons associée à la transition.

Il existe par contre toute une classe de systèmes métalliques dans lesquels on observe des instabilités structurales dites de Peierls, où la position de l'instabilité dans l'espace réciproque est reliée à la géométrie de la surface de Fermi des électrons et peut donc être commensurable ou incommensurable suivant la topologie des bandes électroniques.

La source d'anharmonicité, dans ce cas, est liée au couplage électron-phonon, lequel donne des effets particulièrement spectaculaires dans les métaux $1 \mathrm{D}$ ou quasi-1D, i.e. dans des systèmes où la conductivité électrique est très anisotrope [40].

Le composé $\mathrm{KCP}\left(\mathrm{K}_{2} \mathrm{Pt}(\mathrm{CN})_{4} \mathrm{Br}_{0.3} \cdot 3 \mathrm{H}_{2} \mathrm{O}\right)$ est un bon exemple de ce type de système. La Fig. 16(a) montre la structure des chaînes de Pt parallèles à l'axe c tétragonal. La bande électronique de conduction est constituée par le recouvrement des orbitales externes $\mathrm{d}_{\mathrm{z}}^{2}$ des ions Pt. L'addition de $0.3 \mathrm{Br}$ par formule, crée des trous dans la bande de conduction, qui sont à l'origine du comportement métallique 1D du composé dans la direction c.
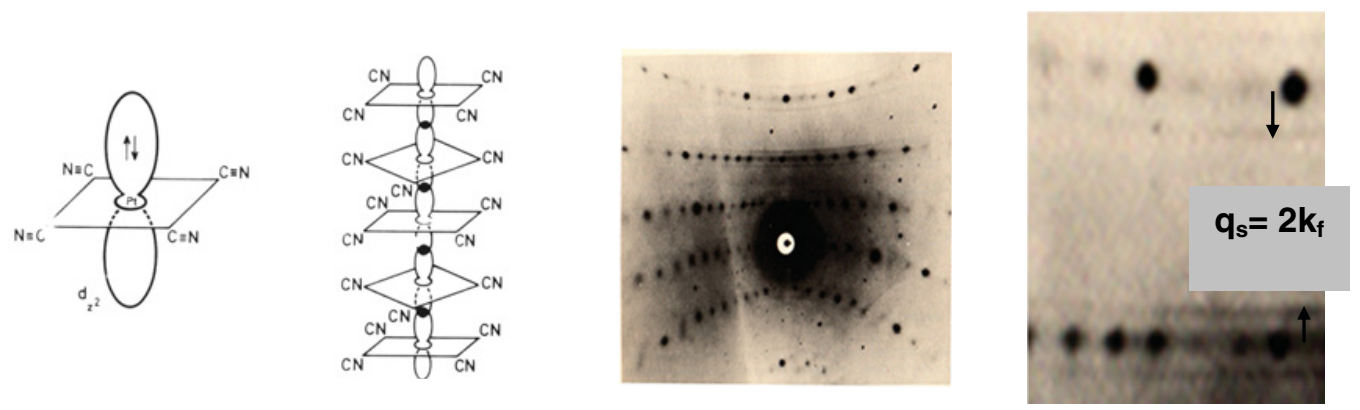

Figure 16. KCP : (a) Structure métallique des chaînes de Pt (d'après Minot et al., réf. [41]) ; (b) Diffusion diffuse des RX : les lignes horizontales continues correspondent à l'anomalie de Kohn 1D sur les chaînes de Pt (d'après Comès et al., réf. [42]) ; (c) Agrandissement de (b) montrant le vecteur d'onde de l'anomalie $\mathrm{q}_{\mathrm{s}}=2 \mathrm{k}_{\mathrm{f}}=0.3 \mathrm{c}^{*}$ (la distance entre strates de Bragg est de $2 \mathrm{c}^{*}$ ).

La surface de Fermi d'une chaîne métallique consiste en 2 plans perpendiculaires à la chaîne, séparés par 2 fois le vecteur de Fermi le long de la chaîne, $2 \mathrm{k}_{\mathrm{f}}$, qui ici vaut $0.3 \mathrm{c}^{*}$ (le même 0.3 que dans la 
formule chimique). Et donc tous les phonons dont les vecteurs d'onde ont une composante égale à 0.3 le long de $\mathrm{c}^{*}$, pourront diffuser un électron d'un feuillet à l'autre de la surface de Fermi.

Il en résulte une anomalie dans la dispersion des phonons, anomalie de Kohn [43], dûe au couplage électron-phonon, avec un caractère 1D très marqué, i.e. qu'on aura des plans de phonons mous d'indice \pm 0.3 le long de $\mathrm{c}^{*}$.

Sur les clichés RX de la Fig. 16(b, c) on distingue des traînées diffuses très fines suivant $\mathrm{c}^{*}$ et très continues, de part et d'autre de chaque strate de taches de Bragg d'indice pair suivant c*. Ces traînées sont les intersections des plans réciproques de phonons mous avec la sphère d'Ewald. L'agrandissement de la Fig. 16(c) permet de vérifier que la distance des traînées diffuses aux strates de taches de Bragg est bien de l'ordre de $0.3 \mathrm{c}^{*}\left(=2 \mathrm{k}_{\mathrm{f}}\right)$. Les strates de Bragg d'indice impair sont très peu visibles du fait de l'existence d'une pseudo-périodicité de la structure, égale à c/2 (= distance Pt-Pt).

Lorsqu'on mesure la dispersion des phonons, en diffusion inélastique des neutrons, on voit en effet une anomalie très marquée sur une nappe de phonons, pour tous les vecteurs d'onde ayant une composante suivant c* égale à 0.3 (Fig. 17(a)). L'anomalie est vue sur la branche LA en partant d'une raie de Bragg, mais aussi lorsqu'on part du point $(1 / 2,1 / 2,0)$ qui est un bord de zone dans le plan de base et qu'on se déplace dans la direction $\mathbf{c}^{*}$. Elle existe également à partir de n'importe quel point du plan de base.

(a)

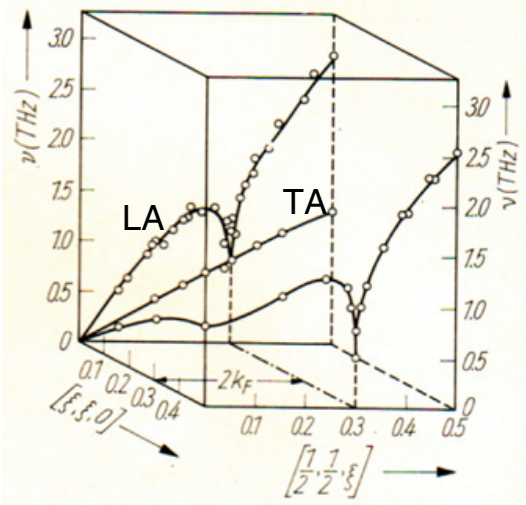

(b)

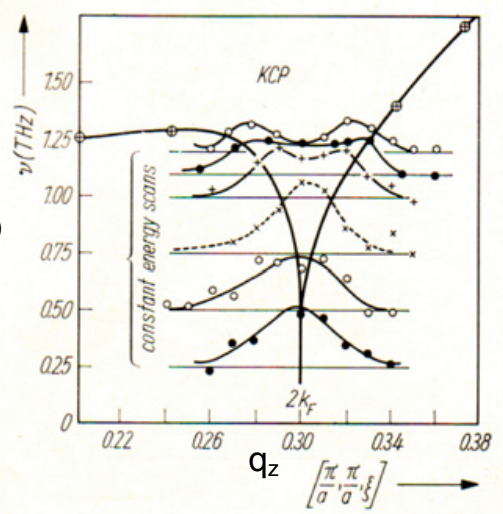

Figure 17. KCP (295 K): (a) Anomalie de Kohn 1D sur le feuillet de dispersion des modes polarisés dans la direction $\mathbf{c}$; les modes polarisés dans le plan de base (TA) ne sont pas affectés; (b) Balayages à énergie constante à travers l'anomalie au point $(1 / 2,1 / 2,0.3)$; d'après Comès et al., réf. [44].

L'anomalie est très étroite dans la direction $\mathbf{c}^{*}$. Des balayages à énergie constante à travers l'anomalie donnent un signal jusqu'à $\omega=0$, mais pour $\omega<3 \mathrm{meV}(0.75 \mathrm{THz})$, on ne sépare plus les deux bords du puits (Fig. 17(b)).

En dessous de la transition de Peierls $\left(\mathrm{T}<\mathrm{T}_{\mathrm{P}} \approx 120 \mathrm{~K}\right)$ les positions des ions Pt le long des chaînes sont modulées statiquement, ainsi que la densité locale des électrons de conduction, on dit qu'on a une Onde de Densité de Charge (ODC) de périodicité $2 \pi / 2 \mathrm{k}_{\mathrm{F}}$ le long des chaînes.

En fait les chaînes sont déjà modulées statiquement ou quasi-statiquement au-dessus de $\mathrm{T}_{\mathrm{P}}$, car l'intensité diffuse a une composante élastique ou quasi-élastique non-résolue $(\omega \approx 0)$ en plus de l'anomalie de phonon. Mais ces ODCs sont déphasées de façon plus ou moins aléatoire les unes par rapport aux autres et c'est ce déphasage aléatoire qui fait que la diffusion diffuse est distribuée de façon uniforme dans des plans réciproques.

Ce qui se passe au voisinage de $\mathrm{T}_{\mathrm{P}}$, c'est la mise en ordre progressive des phases des ODCs, via le développement des corrélations transverses. L'ordre choisi est un ordre AF $(1 / 2,1 / 2,0.3)$, et ce pour au 
moins 2 raisons : d'abord pour des raisons de répulsion coulombienne entre les ODCs, et ensuite parce que les surfaces de Fermi ne sont pas complètement planes. Elles sont légèrement gondolées du fait du tunneling des électrons d'une chaîne à l'autre, ce qui a pour conséquence que certains phonons donnent un meilleur nesting d'un feuillet sur l'autre et dans les cas simples on peut montrer que c'est les phonons de bord de zone qui sont les plus efficaces de ce point de vue [45].

On voit donc que, une fois encore, l'anisotropie du système confère à la dynamique de la transition un caractère mixte, displacif dans la direction des chaînes et plutôt $\mathrm{O} / \mathrm{D}$ dans les directions transverses.

\subsubsection{Cristaux moléculaires : le cas du biphényl}

Les cristaux moléculaires forment une autre grande catégorie de solides, caractérisée par des potentiels interatomiques de nature très différente du cas des cristaux ioniques ou métalliques. Ils sont le siège d'une grande diversité de transitions de phase structurales, conduisant à des phases ordonnées de type $\mathrm{F}, \mathrm{AF}$ ou incommensurables. Les transitions à caractère displacif impliquent des modes de vibrations du type modes externes (i.e. des translations ou des rotations de molécules), comme pour le chloranil, $\mathrm{C}_{6} \mathrm{Cl}_{4} \mathrm{O}_{2}$ [46,47], ou des modes internes comme pour le biphényl, $\mathrm{C}_{12} \mathrm{H}_{10}$ [48].

Dans le cas du biphényl, le mode impliqué est une rotation de type twist des 2 noyaux benzéniques de la molécule. À l'état libre, les plans des 2 noyaux font entre eux un angle de $40^{\circ}$, alors que dans le cristal les 2 noyaux sont coplanaires, afin de permettre un empilement plus compact des molécules. A plus basse température, les amplitudes de vibration diminuant, la place libre pour les molécules augmente et celles-ci en ' profitent' pour revenir à une configuration non-coplanaire, avec la condensation d' une déformation de type twist pour $\mathrm{T}<\mathrm{T}_{\mathrm{I}}=38 \mathrm{~K}$. On a donc une situation analogue au modèle des doublepuits couplés, avec compétition entre un potentiel local (intramoléculaire) et un potentiel interparticule, lié à l'encombrement stérique entre molécules voisines $[49,50]$.

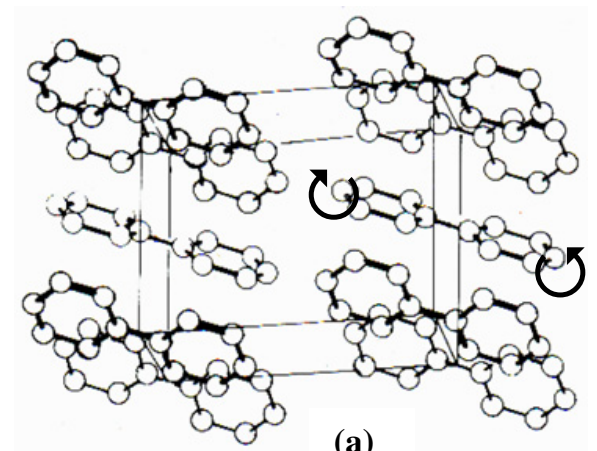

(a)

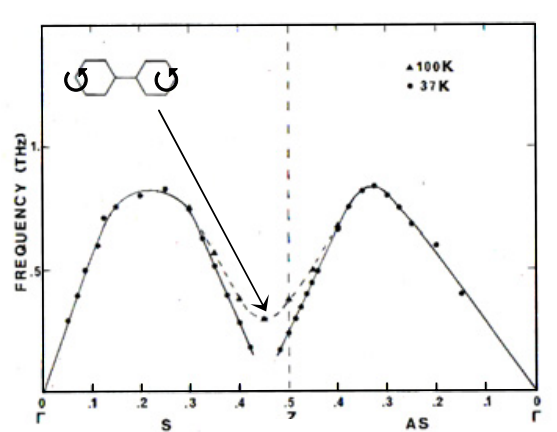

(b)

Figure 18. Biphényl : (a) structure cristalline $(\mathrm{T}>38 \mathrm{~K})$; les flèches circulaires symbolisent le mode de torsion intramoléculaire ; (b) dispersion du mode de torsion dans la direction $\mathbf{b}^{*}$; d'après Cailleau, réf. [48].

En diffusion inélastique des neutrons, on observe le ramollissement du mode de torsion intramoléculaire au voisinage du pt $(0,1 / 2,0)$ de la zone de Brillouin monoclinique, mais la présence d'un invariant de Lifshitz en ce point, fait que le mode se condense en des positions incommensurables, triplement incommensurable au départ, et incommensurable suivant $\mathbf{b}^{*}$ seulement, en dessous de $\mathrm{T}_{\mathrm{II}}=20 \mathrm{~K}$ (transition de lock-in partiel). 


\section{FONCTION-RÉPONSE : MODE MOU ET PIC CENTRAL}

\subsection{Phonon mou suramorti}

Jusqu'à présent nous n'avons rien dit sur la forme de raie, i.e. sur la fonction-réponse associée au mode mou, telle qu'elle apparaît dans une mesure de diffusion inélastique, des neutrons, par exemple. De façon standard, la fonction-réponse, ou susceptibilité linéaire $\chi(\omega, \mathrm{T})$, associée à un oscillateur harmonique amorti s'écrit sous la forme :

$$
\chi(\omega, \mathrm{T})=\left\{\omega_{T}^{2}-\mathrm{i} \omega \Gamma_{\mathrm{T}}-\omega^{2}\right\}^{-1}
$$

où $\omega_{\mathrm{T}}$ et $\Gamma_{\mathrm{T}}$ sont, respectivement, la fréquence propre renormalisée et le coefficient d'amortissement de l'oscillateur, à la température T. Cette formulation est applicable au cas des phonons en présence d'interactions anharmoniques phonon-phonon faibles. Sa validité dans le cas du phonon mou n'est donc pas garantie [20].

Mis à part les facteurs d'origine instumentale [51], le signal mesuré en diffusion inélastique des neutrons est donné par la quantité :

$$
\mathrm{S}_{\mathrm{ph}}(\mathbf{Q}, \omega)=\pi^{-1}\{1-\exp (-\hbar \omega / \mathrm{kT})\}^{-1}|\mathrm{~F}(\mathbf{Q}, \mathbf{q})|^{2} \operatorname{Im} \chi(\omega, \mathrm{T})
$$

où le facteur de structure inélastique du phonon $\mathrm{F}(\mathbf{Q}, \mathbf{q})$ s'exprime, comme une somme sur les $\mathrm{r}$ atomes de la maille $(\kappa=1, \mathrm{r})$ :

$$
\mathrm{F}(\mathbf{Q}, \mathbf{q})=\sum_{\kappa} \mathrm{b}_{\kappa}\left(\mathrm{m}_{\kappa}\right)^{-1 / 2}\left\{\mathbf{Q} \cdot \mathbf{e}_{\kappa}(\mathbf{q})\right\} \exp \left(\mathrm{i} \mathbf{Q} \cdot \mathbf{r}_{\kappa}\right) \exp \left\{-\mathrm{W}_{\kappa}(\mathbf{Q})\right\}
$$

Les quantités $b_{\kappa}, m_{\kappa}, r_{\kappa}, W_{\kappa}, \mathbf{e}_{\kappa}$ sont, respectivement, les longueurs de diffusion cohérente, les masses, les positions et les coefficients de Debye-Waller atomiques, et les vecteurs propres du mode.

En combinant (5.1) et (5.2), on obtient, dans l'approximation classique ( $\hbar \omega \ll \mathrm{kT})$ :

$$
\mathrm{S}_{\mathrm{ph}}(\mathbf{Q}, \omega)=\mathrm{kT} / \hbar \pi|F(\mathbf{Q}, \mathbf{q})|^{2} \Gamma_{\mathrm{T}}\left\{\left(\omega^{2}-\omega_{\mathrm{T}}^{2}\right)^{2}+\omega^{2} \Gamma_{\mathrm{T}}^{2}\right\}^{-1}
$$

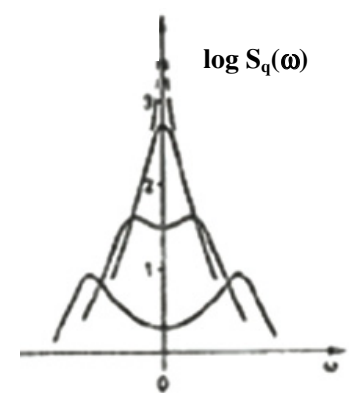

Figure 19. Fonction-réponse d'un oscillateur en fonction du taux d'amortissement $\Gamma_{\mathrm{T}} / \omega_{\mathrm{T}}$. La réponse se concentre de plus en plus autour de $\omega=0$, à mesure que le taux d'amortissement augmente. Si $\omega_{T} \rightarrow 0$ avec $\Gamma_{\mathrm{T}}$ constant (cas du mode mou), la réponse intégrée en fréquence (i.e. la susceptibilité statique) diverge comme $\omega_{\mathrm{T}}^{-2}$. On notera l'échelle verticale logarithmique.

À l'approche de $\mathrm{T}_{c}$, la fréquence propre $\omega_{T}$ diminue tandis que l'amortissement $\Gamma_{\mathrm{T}}$ ne présente pas de comportement critique et peut donc être supposé constant en première approximation. Dans ces conditions, on s'attend à voir la réponse du mode mou évoluer comme indiqué schématiquement en Fig. 19 , avec passage d'un profil de raie à 2 maxima $\left(\omega \approx \pm \omega_{\mathrm{T}}\right)$ vers un profil à un seul pic centré sur $\omega=0$. 
Par ailleurs l'intensité intégrée en fréquence diverge puisque, d'après Kramers-Kronig :

$$
\int \operatorname{Im} \chi(\omega, \mathrm{T}) \omega^{-1} \mathrm{~d} \omega=\chi(\omega=0, \mathrm{~T}) \omega_{\mathrm{T}}^{-2}
$$

Dans la limite très suramortie $\left(\omega_{\mathrm{T}} \ll \Gamma_{\mathrm{T}}\right)$, la fonction-réponse devient Lorentzienne :

$$
\operatorname{Im} \chi(\omega, \mathrm{T})=\pi^{-1} \frac{\omega \gamma_{\mathrm{T}}}{\omega_{\mathrm{T}}^{2}\left(\omega^{2}+\gamma_{\mathrm{T}}^{2}\right)} ; \quad \gamma_{\mathrm{T}}=\omega_{\mathrm{T}}^{2} / \Gamma_{\mathrm{T}} \ll \omega_{\mathrm{T}}
$$

avec une largeur $\gamma_{\mathrm{T}}$ qui tend vers 0 comme $\omega_{\mathrm{T}}^{2}$.

Une Lorentzienne dont la largeur tend vers 0 , voilà qui rappelle beaucoup ce qu'on observe à l'approche d'une transition du type $\mathrm{O} / \mathrm{D}$, sauf que dans un vrai système $\mathrm{O} / \mathrm{D}$ la dynamique est relaxationnelle à toute température. Ici on a un crossover entre une dynamique displacive, de type phonon mou, loin de $T_{c}$, et une dynamique relaxationnelle près de $T_{c}$. Faut-il en conclure que, suffisamment près de $T_{c}$, tout système displacif se comporte comme un système $O / D$ ?

\subsection{Pic central}

En fait, l'expérience montre que les choses ne se passent pas de cette manière et que le régime très suramorti n'est pas observé dans les systèmes réels. La Fig. 20(a) montre la forme de raie observée dans le cas du chloranil [47]. Le chloranil $\left(\mathrm{C}_{6} \mathrm{Cl}_{4} \mathrm{O}_{2}\right)$ est un cristal moléculaire formé de molécules de type cycle benzénique. Vers $100 \mathrm{~K}$ on observe des rotations moléculaires alternées d'amplitude croissante avec apparition de raies de surstructure en dessous de $\mathrm{T}_{\mathrm{c}} \approx 90 \mathrm{~K}$.
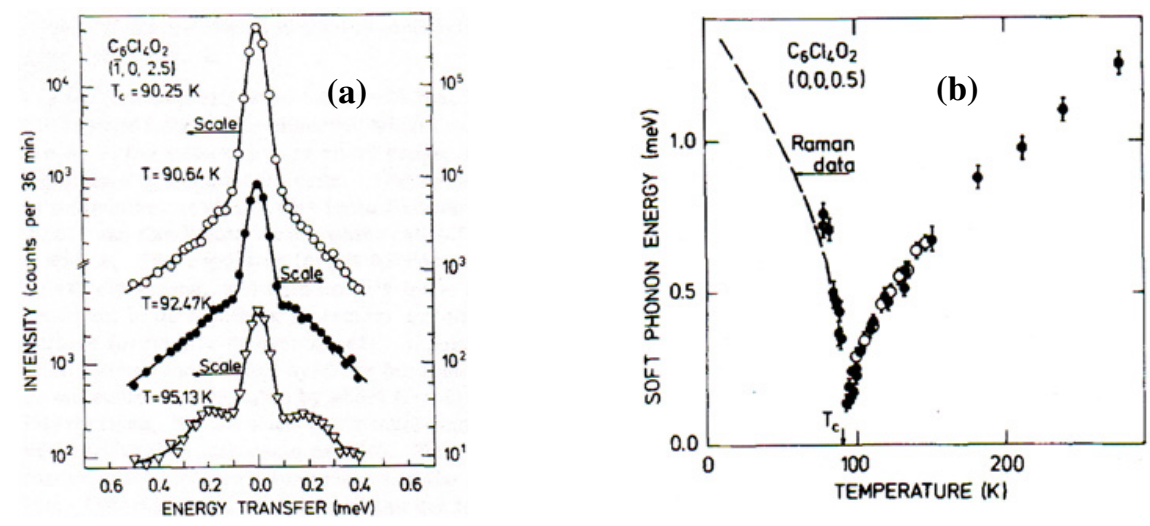

Figure 20. Chloranil $\left(\mathrm{C}_{6} \mathrm{Cl}_{4} \mathrm{O}_{2}\right)$ : (a) Analyse en énergie de la diffusion critique, montrant la coexistence du phonon mou et du pic central $(\omega \approx 0)$; les échelles d'intensité sont logarithmiques; (b) Fréquence propre du mode mou, $\omega_{\mathrm{T}} ;$ d'aprés Ellenson and Kjems, réf. [47].

En plus du phonon mou qui devient progressivement suramorti à l'approche de $\mathrm{T}_{\mathrm{c}}$, la Fig. 20(a) montre l'existence $(\mathrm{T}<140 \mathrm{~K}$ ) d'une composante élastique (ou quasi-élastique, non résolue en fréquence), le fameux pic central (PC), dont l'intensité croît continûment et diverge à $\mathrm{T}_{\mathrm{c}}$.

Par ailleurs, l'analyse des spectres montre que la fréquence propre du phonon mou, $\omega_{\mathrm{T}}$, tend vers une valeur finie à $\mathrm{T}_{\mathrm{c}}$ (Fig. 20(b)). En dessous de $\mathrm{T}_{\mathrm{c}}$, la fréquence du phonon mou remonte et la raie centrale se confond avec la raie (élastique) de surstructure, qui apparaît à $\mathrm{T}_{\mathrm{c}}$ et croît très vite en dessous (voir Fig. 11 et discussion au \$4.2.1).

On a donc bien là, une dynamique à 2 échelles, comme on pourrait s'attendre à en rencontrer dans les cas intermédiaires displacif-O/D. Il est, en effet, tentant d'associer la largeur en q et la largeur en fréquence du PC à la taille et au temps de vie moyens de régions quasi-statiquement «ordonnées » 
(clusters). Par ailleurs, à l'intérieur des clusters, on s'attend à ce que la dynamique vibrationnelle soit analogue à ce qui est observé lorsque le cristal est macroscopiquement ordonné $\left(\mathrm{T}<\mathrm{T}_{\mathrm{c}}\right)$, On sait alors que la fréquence du mode mou augmente (voir Figs. 13 et 20(b)). On comprend alors que l'apparition des clusters, à l'approche de $\mathrm{T}_{c}$, ait pour effet de stabiliser la fréquence du phonon mou et de lui donner une valeur limite finie à $\mathrm{T}_{\mathrm{c}}$.

Le PC a été d'abord mis en évidence dans $\mathrm{SrTiO}_{3}$ [52,53] et on s'est aperçu ensuite que le même phénomène existait, plus ou moins, au voisinage de toutes les transitions de phase displacives.

Cette découverte a provoqué un véritable engouement théorique pour essayer de rendre compte de la formation de ces «clusters ordonnés » au-dessus de $\mathrm{T}_{\mathrm{c}}$, sur la base de modèles anharmoniques non-linéaires plus ou moins sophistiqués [54], en gros, en essayant d'aller au-delà du modèle un peu simpliste du mode mou.

Le problème c'est que personne n'a été capable de mesurer la largeur en fréquence du PC, par quelque technique que ce soit. Dans le cas des mesures TAS sur le chloranil (Fig. 20(a)), le PC a, à toutes températures, la largeur instrumentale $(0.14 \mathrm{meV}=35 \mathrm{GHz})$. Des résultats analogues ont été obtenus dans le cas de $\mathrm{SrTiO}_{3}$ et $\mathrm{KMnF}_{3}$ [53], malgré des conditions expérimentales optimisées (neutrons froids, collimations : $4 \times 20^{\prime}$ ). D'autres tentatives, utilisant d'autres techniques neutroniques (back-scattering [55], spin-echo [56]) n'ont pu mettre en évidence aucune largeur intrinsèque, même loin de $\mathrm{T}_{\mathrm{c}}$.

De plus, l'étude de la forme de la raie de résonance RPE, par le groupe de K. A. Müller à IBM-Zürich [57] a permis de mettre une limite supérieure à la largeur en fréquence du PC, de l'ordre du MHz. Des expériences en spectroscopie Mössbauer par Darlington et O'Connor [58] donnent également une limite supérieure de quelques MHz.

Il est clair que pour faire «sortir du chapeau » une fréquence caractéristique de l'ordre du MHz, en partant d'un champ de phonons anharmoniques dont les fréquences propres et les largeurs de raie sont toutes dans la gamme de $100 \mathrm{GHz}$ ou plus, il faut avoir beaucoup de talent, et la plupart des théoriciens ont fini par se décourager.

Toutes ces théories dites intrinsèques, prédisent en effet que, au moment où le phénomène apparaît, 15-20 K au-dessus de $\mathrm{T}_{c}$, dans le cas de $\mathrm{SrTiO}_{3}$, le $\mathrm{PC}$ devrait avoir une largeur mesurable, de l'ordre des fréquences caractéristiques de phonons, or ce n'est pas le cas, comme on l'a vu plus haut.

Des modèles qui semblent plus plausibles $[59,60]$ font appel au rôle que peuvent jouer certains défauts ou impuretés qui ont leur propre dynamique avec des temps de relaxation dans la gamme du $\mathrm{MHz}$ (ou plus lent), et dont la géométrie permet d'imaginer un couplage linéaire avec les déplacements atomiques associés au mode mou. Dans le cas de $\mathrm{SrTiO}_{3}$, on peut penser à une molécule diatomique en position interstitielle, ou, d'une manière générale, à tout ce qui peut favoriser une rotation locale des octaèdres dans un sens plutôt que dans l'autre.

La manière la plus simple de décrire ce couplage, phénoménologiquement, est d'inclure un relaxeur de Debye dans la fonction d'amortissement du mode mou :

$$
\mathrm{i} \omega \Gamma_{\mathrm{T}} \Rightarrow \mathrm{i} \omega \Gamma_{\mathrm{T}}+\delta^{2} /(1-\mathrm{i} \omega \tau)
$$

où $\delta$ représente la constante de couplage phonon mou-relaxeur et $\tau$ le temps de relaxation propre du défaut.

En introduisant (5.7) dans (5.1), on obtient l'expression de la susceptibilité du mode mou « couplé »:

$$
\chi_{\text {coupl }}(\omega, \mathrm{T})=\left\{\omega_{\mathrm{T}}^{2}-\mathrm{i} \omega \Gamma_{\mathrm{T}}-\delta^{2} /\left(1-\mathrm{i} \omega \tau-\omega^{2}\right\}^{-1}\right.
$$

et on montre que [53], sous certaines conditions, le profil de raie se décompose alors en un oscillateur de fréquence propre $\omega_{\mathrm{T}}$, et une composante quasi-élastique de largeur $1 / \tau^{\prime}=(1 / \tau)\left(\omega_{\mathrm{T}}^{2}-\delta^{2}\right) / \omega_{\mathrm{T}}^{2}$. La température de transition est donnée par la divergence de la susceptibilité statique couplée :

$$
\left\{\chi_{\text {coupl }}\left(0, \mathrm{~T}_{c}\right)\right\}^{-1}=\omega_{\mathrm{Tc}}^{2}-\delta^{2}=0
$$


ce qui implique qu'à $\mathrm{T}_{c}$ la fréquence du phonon reste finie et égale à la constante de couplage $\delta$. Un petit calcul montre également que l'intensité intégrée du PC est donnée par :

$$
\mathrm{I}_{\mathrm{PC}}=\delta^{2} / \omega_{\mathrm{T}}^{2}\left(\omega_{\mathrm{T}}^{2}-\delta^{2}\right)
$$

et qu' elle diverge donc à $\mathrm{T}_{\mathrm{c}}$ comme $\left(\omega_{\mathrm{T}}^{2}-\delta^{2}\right)^{-1}$.

L'idée générale qui se dégage de ce modèle, c'est que la transition s'effectue par un processus de nucléation de régions ordonnées centrées sur des défauts couplés linéairement aux fluctuations critiques, et qui donc favorisent localement l'apparition d'un des domaines de la phase ordonnée. Et qu'ensuite ces micro-domaines croissent au dépend les uns des autres, par la relaxation des défauts, de façon à parvenir à une mise en ordre du système à une échelle macroscopique.

Le point faible de ce type de modèle est que, malheureusement, la nature microscopique des défauts actifs n'a pas été clairement identifiée, à ce jour.

\section{CONCLUSION}

Le but de cet article est de rappeler les notions de base (symétries brisées, paramètre d'ordre, fluctuations critiques, longueurs de corrélation, etc...) qui jouent un rôle central dans l'étude des transitions de phase en général, et d'exposer leur contenu physique, dans le cas des transitions structurales. Ce travail s'adresse à ceux qui désirent se familiariser ' de loin ' avec le sujet, essentiellement dans le but d'élargir leur culture générale. À ceux qui voudraient s'y intéresser de façon plus précise et qui sont prêts à y investir un peu plus de temps, je recommanderais la lecture des articles de revues de Cowley [20] et Bruce [13].

Les transitions de phase structurales ont connu un développement intense pendant les années 60-80, période au cours de laquelle la quasi-totalité des concepts et des résultats expérimentaux importants ont été acquis. La diffusion inélastique des neutrons a joué un rôle majeur dans ce développement et réciproquement, les progrès en matière d'instrumentation neutronique ont souvent été stimulés par les défis posés par l'étude des transitions de phase. Il reste cependant un grand nombre de questions ouvertes, notamment pour tout ce qui touche au rôle des contraintes et des défauts cristallins et c'est là un aspect important qui distingue les transitions structurales des transitions magnétiques.

Les transitions structurales restent un sujet actuel, pour plusieurs raisons. D'abord parce que les progrès en matière de techniques expérimentales permettent occasionnellement de «revisiter» des problèmes non résolus [61-65]. Et ensuite parce que les «nouveaux » matériaux s'avèrent aussi riches en instabilités structurales de toutes sortes, que les autres et il est toujours souhaitable, même si cela ne constitue pas un objet de recherche en soi, de savoir identifier les mécanismes qui donnent lieu à ces changements de structure, ne serait-ce que pour mieux comprendre en quoi ils peuvent affecter les propriétés «utiles » des dits matériaux.

\section{Références}

[1] G.A. Samara,T. Sakudo and K. Yoshimitsu, Phys. Rev. Lett. 35 (1975) 1767.

[2] L. D. Landau, Phys. Z. Sowjun. 11 (1937) 26 ; L. D. Landau and E. M. Lifshitz : Statistical Physics Chap. 14 (Pergamon Press, London, 1968).

[3] E. L. Wagner and D. F. Hornig, J. Chem. Phys. 18 (1950) 296 ; H. A. Levy and S. M. Peterson, J. Am. Chem. Soc. 75 (1953) 1536.

[4] Y. Yamada, Y. Noda, J. D. Axe and G. Shirane, Phys. Rev. B 9 (1974) 4429 ; Y. Yamada, H. Takatera and L. Huber, J. Phys. Soc. Jpn 36 (1974) 641.

[5] J. Töpler, D. R. Richter and T. Springer, J. Chem. Phys. 69 (1978) 3170.

[6] G. Coddens, J. Phys. IV France 10 (2000) Pr1-151.

[7] M. Born and K. Huang : Dynamical Theory of Crystal Lattices (Clarendon Press, Oxford, 1962).

[8] D. Strauch and B. Dorner, J. Phys. C : Solid State Phys. 19 (1986) 2853. 
[9] W. Cochran, Adv. Phys. 9 (1960) 387.

[10] P. W. Anderson, p. 290 in Fizika Dielectrikov, G. I. Skanavi ed. (Acad. Nauk SSSR, Moscow, 1960).

[11] S. Aubry and R. Pick, Proceed. IMF3, Edinburg, 1973 [Ferroelectrics 8 (1974) 585] ; S. Aubry, J. Chem. Phys. 62 (1975) 3217; 64 (1976) 3392.

[12] J. A. Krumhansl and J. R. Schrieffer, Phys. Rev. B 11 (1975) 3535.

[13] A. D. Bruce, Adv. Phys. 29 (1980) 111.

[14] R. Comès, M. Lambert and A. Guinier, Acta Cryst. A 26 (1970) 244.

[15] J. H. Barrett, Phys. Rev. 86 (1952) 118.

[16] J. D. Axe, J. Harada and G. Shirane, Phys. Rev. B 1 (1970) 1227

[17] E. Farhi, A. K. Tagantsev, R. Currat, B. Hehlen, E. Courtens and L. A. Boatner, Eur. Phys. B 15 (2000) 615.

[18] H. Voigt, Ferroelectrics 184 (1996) 31.

[19] R. H. Lyddane, R. G. Sachs and E. Teller, Phys. Rev. 59 (1941) 673.

[20] R. A. Cowley, Adv. Phys. 29 (1980) 1.

[21] G. Shirane, R. Nathans and V. J. Minkiewicz, Phys. Rev. 157(1967) 396.

[22] R. L. Migoni, H. Bilz and D. Baüerle, Phys. Rev. Lett. 37 (1976) 1155.

[23] R. A. Cowley, Phil. Trans. R. Soc. London A 354 (1996) 2799.

[24] B. Okai and J. Yoshimoto , J. Phys. Soc. Jpn. 39 (1975) 162.

[25] G. Shirane, V. J. Minkiewicz and A. Linz, Solid. St. Commun. 8 (1970) 1941.

[26] K. A. Müller, E. Brun, B. Derighetti, J. E. Drumheller and F. Waldner, Phys. Letters 9 (1964) 223.

[27] E. S. Kirkpatrick, K. A. Müller and R.S. Rubins, Phys. Rev. 135 (1964) A86.

[28] A. D. Bruce and A. Aharony, Phys. Rev. B 11 (1975) 478.

[29] T. Riste, E.J. Samuelson and K. Otnes in Structural Phase Transitions and Soft Modes, eds. E.J. Samuelson, E. Anderson and J. Feder (Oslo Universitetsforlaget, 1971), pp. 395-408.

[30] K. A. Müller and W. Berlinger, Phys. Rev. Lett. 26 (1971) 13.

[31] G. Shirane and Y. Yamada, Phys. Rev. 177 (1969) 858.

[32] R. A. Cowley, W. J. L. Buyers and G. Dolling, Solid. St. Commun. 7 (1969) 181.

[33] P. A. Fleury, J. F. Scott and J. M. Worlock, Phys. Rev. Lett. 21 (1968) 16.

[34] J. Feder and E. Pytte, Phys. Rev. B 1 (1970) 4803.

[35] W. D. Johnston, Jr and I. P. Kaminow, Phys. Rev. 168 (1968) 1045.

[36] Light Scattering near Phase Transitions, eds. H. Z. Cummins and P. Levanyuk (North-Holland, Amsterdam, 1983)

[37] J. D. Axe, M. Iizumi and G. Shirane, Phys. Rev. B 22 (1980) 3408.

[38] Y. Ishibashi, Ferroelectrics 20 (1978) 103.

[39] M. Iizumi, J. D. Axe, G. Shirane and K. Shimaoka, Phys. Rev. B 15 (1977) 4392.

[40] G. Grüner, Rev. Mod. Phys. 60 (1988) 1129.

[41] M. J. Minot and J. H. Perlstein, Phys. Rev. Lett. 26 (1971) 371.

[42] R. Comès, M. Lambert, H. Launois and H.R. Zeller, Phys. Rev. B 8 (1973) 571.

[43] W. Kohn, Phys. Rev. Lett. 2 (1959) 393.

[44] R. Comès, B. Renker, L. Pintschovius, R. Currat, W. Gläser and G. Scheiber, Phys. Stat. Sol. 71 (1975) 171.

[45] S. Barisic, Phys. Rev. B 5 (1972) 941.

[46] H. Terauchi, T. Sakai and H. Chihara, J. Chem. Phys. 62 (1975) 383.

[47] W. D. Ellenson and J. K. Kjems, J. Chem. Phys. 67 (1977) 3619.

[48] H. Cailleau in Incommensurate Phases in Dielectrics, eds. R. Blinc and A. P.Levanyuk, Vol. 2, pp. 72-100. North-Holland, Amsterdam, 1986.

[49] V. Heine and S. L. Price, J. Phys. C 18 (1985) 5259.

[50] T. Janssen, Jpn. J. App. Phys. 24 (Suppl. 2) (1985) 747. 
[51] B. Hennion, this volume; R. Currat in Neutron and X-ray Spectroscopy, eds. F. Hippert et al., pp. 383-425. Springer, The Netherlands, 2006.

[52] T. Riste, E. J. Samuelsen, K. Otnes and J.K Feder, Solid St. Comm. 9 (1971) 1455.

[53] S. M. Shapiro, J. D. Axe, G. Shirane and T. Riste, Phys. Rev. B 6 (1972) 4332.

[54] Anharmonic Lattices, Structural Phase Transitions and Melting, ed. T. Riste (Noordhoff-Leiden, Groningen, 1974). F. Schwabl, p. 87 ; J. Feder, p. 113.

[55] J. Töpler, B. Alefeld and A. Heidemann, J. Phys. C 10 (1977) 635.

[56] F. Mezei and J. Hayter, EPS Conference, Budapest, 1974 (unpublished).

[57] G. F. Reiter, W. Berlinger, K. A. Müller and P. Heller, Phys. Rev. B 21 (1980) 1.

[58] C. N. W. Darlington and D. A. O’Connor, J. Phys. C 9 (1976) 3561.

[59] B. I. Halperin and C. M. Varma, Phys. Rev. B 14 (1976) 4030.

[60] A. P. Levanyuk, V. V. Osipov and A. A. Sobyanin, in Theory of Light Scattering in Condensed Matter, eds. B. Bendow, J. L. Birman and V. M. Agranovich (Plenum, New York, 1976) p. 517.

[61] J. P. Itié, B. Couzinet, A. Polian, A. M. Flank and P. Lagarde, Europhys. Lett. 44 (2006) 706.

[62] B. Zalar, A. Lebar, J. Seliger, R. Blinc, V. V. Laguta and M. Itoh, Phys. Rev. B 71 (2005) 64107.

[63] S. Ravy, J. P. Itié, A. Polian and M. Hanfland, Phys. Rev. Lett. 99 (2007) 117601.

[64] B.D. Chapman, E. A. Stern, S. W. Han, J. O. Cross, G. T. Seidler, V. Gavrilytchenko, R. V. Vedrinskii and V. L. Kraizman, Phys. Rev. B 71 (2005) 020102(R).

[65] S. Ravy, D. Le Bolloc'h, R. Currat, A. Fluerasu, C. Mocuta and B. Dkhil, Phys. Rev. Lett. 98 (2007) 105501. 